\title{
Anlotinib Inhibits the Growth of Breast Cancer Cells by Promoting Autophagy and Apoptosis via the Akt/GSK-3a Signalling Pathway
}

Shuyi Chen ( $17111010074 @ f u d a n . e d u . c n)$

Fudan University School of Basic Medical Sciences https://orcid.org/0000-0002-8353-5240 ping zhu

Fudan University

xue wang

Fudan University

youping jin

Fudan University

xiuling zhi

Fudan University

Huanjun Yang

Fudan University

ping zhou

Fudan University

Primary research

Keywords: Anlotinib, breast cancer, autophagy, apoptosis, proliferation

Posted Date: December 2nd, 2020

DOl: https://doi.org/10.21203/rs.3.rs-113481/v1

License: (9) (i) This work is licensed under a Creative Commons Attribution 4.0 International License.

Read Full License 


\section{Abstract}

Background: Anlotinib, a multi-target tyrosine kinase inhibitor, has already been indicated to have significant anticancer effects on lung cancer, colon cancer and ovarian cancer in a phase II clinical trial, but its effect on breast cancer (BC) has not been adequately investigated.

Methods: The proliferation activity of BC cell lines MCF-7 and MDA-MB-231 with the treatment of anlotinib was tested by Cell Counting Kit-8 (CCK-8) assay and immunocytochemistry (ICC) staining. We investigated the alteration of cell cycle and apoptosis and autophagy level and the underlying mechanism in the cell lines by quantitative real-time reverse-transcription polymerase chain reaction (qRTPCR), Western blots, ICC and TUNEL staining and flow cytometry. Further, AT-3 cells were subcutaneously injected into C57BL/6 mice, followed by anlotinib intragastrically. The extracted tumours were assessed by qRT-PCR, Western blots and immunohistochemistry.

Results: We found that anlotinib suppressed the cell viability and proliferation of MCF-7 and MDA-MB231 cell lines and tumour growth in BC xenografts in mice, likely due to abnormal cell cycle arrest and induction of autophagy and apoptosis. Then, we further examined the underlying mechanism of anlotinib, and the results indicated that anlotinib induced apoptosis by promoting autophagy in MCF-7 and MDA-MB-231 cells by regulating the Akt/GSK-3a pathway. The analysis of data from patients with $\mathrm{BC}$ collected in TCGA revealed that increased VEGFA expression was related to BC.

Conclusions: Our study demonstrated that anlotinib inhibited the growth of BC cells via promoting apoptosis through autophagy mediated by Akt/GSK-3a signalling and may be an effective new drug for $\mathrm{BC}$ treatment.

\section{Introduction}

Breast cancer $(\mathrm{BC})$ is one of the most common and high-risk female malignant tumours worldwide. Its incidence rises each year, and the age of onset tends to be younger. The world's leading academic journal "CA: A Cancer Journal for Clinicians" published the latest report of cancer statistics in the United States in 2019 , which indicated that BC ranked first in new cancer cases in women $(30 \%)$ and second in new cancer deaths in women (15\%) in 2019 ${ }^{1}$. In January 2019, China's National Cancer Centre released the latest cancer data in China, which indicated that the incidence of $\mathrm{BC}$ continues to rank first among cancers in women ${ }^{2}$. Although the treatment of BC has improved in recent years, postoperative tumour cell recurrence, metastasis and drug resistance have resulted in low tumour-free survival and 5-year survival rates in high-risk populations, which seriously threaten the lives and health of patients ${ }^{2-4}$. Targeted therapy, as a new therapeutic strategy, has the advantages of strong specificity, remarkable curative effects and few side effects. Hence, targeted therapy has been recognized as an effective and selective method to kill tumour cells, and it is gradually becoming a hot spot and trend in the field of cancer therapy 
Anlotinib hydrochloride, a novel oral multi-target tyrosine kinase receptor inhibitor that targets vascular endothelial growth factor receptor (VEGFR), fibroblast growth factor receptor (FGFR) and platelet-derived growth factor receptor (PDGFR) shows broad-spectrum inhibitory effects on tumour angiogenesis and growth ${ }^{5,6}$. The VEGF subtype and its receptor VEGFR are key proteins in angiogenesis and growth and have been shown to be effective anticancer targets ${ }^{7-9}$. In vitro studies have shown that anlotinib selectively inhibits VEGFR2/kinase insert domain receptor (KDR) and VEGFR3, with an inhibition rate 20 times that of sunitinib and 500 times that of sorafenib, respectively. In addition, the dysregulated FGF/FGFR axis promotes cancer progression and enhances the angiogenic potential of the tumour microenvironment, leading to an invasive phenotype of cancer cells ${ }^{10-15}$, and FGF/FGFR signalling changes are associated with chemoresistance and adverse clinical prognosis of cancers. Preclinical results show that anlotinib inactivates FGFR1-4, especially FGFR2, compared with the effects of sorafenib. Recently, it was found that anlotinib also inhibits PDGFRa/ $\beta$, c-Kit receptor, glial cell-derived neurotrophic factor receptor tyrosine kinase, Aurora-B kinase, c-Fms kinase and discoid domain receptor 1. These receptors or kinases are involved in the proliferation of colon cancer cells, lymphoma cells and acute $T$ cell leukaemia cells or in the progression of lung, breast and ovarian cancers ${ }^{16-19}$. In vivo experiments demonstrated that anlotinib has broad inhibitory effects against xenograft tumours from transplanted human tumour cells, such as colon cancer cells (SW-620), ovarian cancer cells (SK-OV-3), kidney cancer cells (Caki-1), glioma cells (U87MG) and non-small cell lung cancer cells (Calu-3) ${ }^{20}$.

Although anlotinib has been proven to have significant anticancer effects on many malignancies, no study has estimated its effects and underlying mechanisms in BC. Here, we first investigated the effect of anlotinib on the growth of $\mathrm{BC}$ cells and elucidated its mechanisms. Therefore, it demonstrated that anlotinib exerted its anticancer effect on BC, providing a new option for targeted therapy of BC.

\section{Materials And Methods}

\subsection{Compounds}

Anlotinib was kindly given as a gift by Chia Tai Tianqing Co., Ltd. (Nanjing, JS, China). Wort and 3methyladenine (3-MA) were purchased from APExBIO (Houston, TX, USA).

\subsection{Database}

To examine the differences in VEGFA expression in normal and BC tissue, as well as the difference in the subtype of BC, we searched the public database UALCAN.

\subsection{Cell culture}

The human BC cell line MCF-7 was cultured in Dulbecco's modified Eagle's medium (DMEM) containing $10 \%$ foetal bovine serum (FBS) and antibiotics (penicillin $100 \mathrm{U} / \mathrm{ml}$ and streptomycin $100 \mathrm{mg} / \mathrm{ml}$ ) in a $37^{\circ} \mathrm{C}$ humidified atmosphere with $5 \% \mathrm{CO}_{2}$. The human BC cell line MDA-MB-231 (NCI-DTP Cat\# MDAMB231, RRID:CVCL_0062) was cultured in Leibovitz's L-15 medium with $10 \% \mathrm{FBS}$ and antibiotics at $37^{\circ} \mathrm{C}$ in 
a free gas exchange environment with atmospheric air. The MCF-7 and MDA-MB-231 cell lines were purchased from the cell bank of the Chinese Academy of Science. AT-3 cells were cultured in Roswell Park Memorial Institute (RPMI) 1640 medium with $10 \%$ FBS and antibiotics in a $37^{\circ} \mathrm{C}$ humidified atmosphere with $5 \% \mathrm{CO}_{2}$. The cells were obtained from Roswell Park Cancer Institute (Buffalo, NY, USA) and were used as a C57BL/ 6 mouse breast carcinoma cell line according to a previous paper ${ }^{21}$.

\subsection{Cell viability assay}

A Cell Counting Kit-8 (CCK-8) assay was used to detect cell viability. Briefly, 10000 cells for MCF-7 cell and 5000 cells for MDA-MB-231 cell per well were seeded in 96-well plates and incubated with anlotinib at various concentrations and for different times. The CCK-8 reagent was then added at a 1:10 dilution and incubated for $1.5 \mathrm{~h}$, and the absorbance at $450 \mathrm{~nm}$ was measured on a microplate reader to calculate the cell viability and IC50.

\subsection{Antibodies}

The antibodies for western blotting, immunocytochemistry and immunohistochemistry were Ki67, BECN1, phospho-AKT S473, and $\beta$-actin from Proteintech (Rosemont, IL, USA); Bak, Cytochrome c, Bcl-2, cleaved Caspase 9, cleaved Caspase 3, Caspase 1, LC3B, phospho-GSK-3a (Ser21), GSK-3a, and AKT from CST (Beverly, MA, USA); and P62, Cyclin D1, PARP1, and cleaved PARP1 from Abcam (Cambridge, Cambs, UK). More details for the antibodies were list in the Supplemental Table 1.

\subsection{Quantitative real-time PCR (qRT-PCR)}

RNA was extracted from the cells or tissues using the TRIzol-trichloromethane-isopropanol method and reverse transcribed into cDNA according to the protocol for the ReverTra Ace qPCR RT Kit (TOYOBO). The products were mixed with SYBR Green, $\mathrm{ddH}_{2} \mathrm{O}$ and primers and added to the PCR plate and analysed. The sequences of the primers are listed in Supplemental Table 2.

\subsection{Western blot analysis}

Protein samples from cancer cells and tissues were resolved by SDS-PAGE (10\% or $12 \%)$, electrotransferred onto Immobilon-P membranes, blocked, and incubated with primary and secondary antibodies. Densitometric quantification of the protein bands was analysed using ImageJ software (RRID:SCR_003070).

\subsection{Flow cytometry}

For apoptosis analysis, $5 \times 10^{5}-1 \times 10^{6}$ cells in the DMSO and anlotinib groups were collected and stained with Annexin V-FITC and PI according to the protocol for the Annexin V-FITC/PI apoptosis detection kit (Vazyme, Nanjing, JS, China). For cell cycle analysis, pre-treated cells were fixed and stained by PI according to the protocols for the cell cycle and Analysis analysis kit (YEASEN, Shanghai, China). The samples were analysed on a flow cytometer (FACSCalibur) and by using ModFit LT software. 


\subsection{Immunocytochemistry (ICC) and TUNEL staining assays}

Cells were fixed in $4 \%$ paraformaldehyde (PFA) for $15 \mathrm{~min}$ and then permeabilized with $0.1 \%$ Triton X-100 for $20 \mathrm{~min}$. After blocking with $5 \%$ goat serum for $2 \mathrm{~h}$, the cells were incubated with primary antibodies overnight at $4{ }^{\circ} \mathrm{C}$. Then, the cells were washed and incubated with secondary antibodies and finally incubated with DAPI for nuclei staining. Epifluorescent images were taken with an Olympus IX81 microscope. For the TUNEL staining assay, cells were fixed with 4\% PFA first and then processed according to the protocol for the TUNEL BrightRed Apoptosis Detection Kit (Vazyme, Nanjing, JS, China).

\subsection{Immunohistochemistry}

Dissected tumour tissues were preserved in $4 \%$ PFA at $4{ }^{\circ} \mathrm{C}$ for $24 \mathrm{~h}$, dehydrated with xylenes and alcohols, and embedded in paraffin. Sections were cut at a thickness of $5 \mu \mathrm{m}$, dewaxed in xylene, rehydrated through decreasing concentrations of ethanol, and washed in PBS. Antigens were unmasked, blocked and incubated with primary antibodies overnight at $4{ }^{\circ} \mathrm{C}$. For immunohistochemistry, the following steps were the same as those for immunocytochemistry, and the sections were incubated with secondary antibodies and DAPI. Images were taken with an Olympus IX81 microscope.

\subsection{Xenograft mouse model}

C57BL/6 mice were maintained in specific pathogen free (SPF) conditions at the Fudan University Animal Experimental Centre. All animal experiments were approved by the Ethics Committee (Approval number: 20190703). AT-3 cells were harvested and resuspended at $1 \times 10^{7}$ cell/ $\mathrm{ml}$, and the $0.1 \mathrm{ml}$ of suspension was subcutaneously injected into the 20 male mice (each group, $n=10$ ). On day 7 after injection, 10 mice randomly were administered $2.5 \mathrm{mg} / \mathrm{kg}$ anlotinib intragastrically once daily for 3 weeks as anlotinib group, while the DMSO group was treated with the same volume of DMSO $(0.25 \%)$. At the determined time points, the mice were sacrificed, and the tumours were removed and measured.

\subsection{Statistical Analysis}

Statistical analysis was performed by Student's t-test for comparisons between the DMSO and anlotinib groups and two-factor Analysis of Variance (ANOVA) for comparisons among the four groups, followed by a subsequent post-hoc test. Growth curves descripted in the CCK-8 assay were analysed with twofactor ANOVA (Treatment $x$ time). The experiments were repeated at least 3 times independently. The data were presented as mean \pm SD and analysed using GraphPad Prism 6.0 (RRID:SCR_002798), and $p<$ 0.05 was considered statistically significant.

\section{Results}

\subsection{Anlotinib suppressed the cell viability and proliferation of MCF-7 and MDA-MB-231 cells}


To investigate whether anlotinib affected cell proliferation, we used a CCK-8 assay to assess cell viability and the half maximal inhibitory concentration (IC50) in MCF-7 and MDA-MB-231 cells. The results showed that anlotinib inhibited cell viability in a dose- and time-dependent manner, and the IC50 values of the two cell lines treated with anlotinib for $4 \mathrm{~h}, 12 \mathrm{~h}, 24 \mathrm{~h}$ and $48 \mathrm{~h}$ are shown in Fig. 1A and 1B. We also performed ICC analysis for Ki67, and the results showed that proliferation of MCF-7 and MDA-MB231 cells decreased with anlotinib administration (Fig. 1A-D, $n=3$ ).

\subsection{Anlotinib regulated the cell cycle in MCF-7 and MDA- MB-231 cells}

To detect whether anlotinib inhibited cell proliferation by modulating cell cycle progression, we examined alterations in the cell cycle and Cyclin D1 expression levels between the DMSO and anlotinib groups. The mRNA and protein levels of Cyclin D1 in MCF-7 and MDA-MB-231 cells were significantly decreased after treatment with anlotinib at $10 \mu \mathrm{M}$ for $12 \mathrm{~h}$, indicating that anlotinib inhibited cell proliferation by regulating the cell cycle in $B C$ cells (Fig. $1 E$ and F). We found that the fraction of G2/M cells increased markedly in anlotinib-treated cells analysed by flow cytometry (Fig. 1G, ${ }^{* *} p<0.01, n=3$ ).

\subsection{Anlotinib induced apoptosis in MCF-7 and MDA-MB-231 cells}

To investigate the effect of anlotinib on cell apoptosis more comprehensively, the cells treated with or without anlotinib were labelled with PI and Annexin V-FITC and analysed by flow cytometry, and the results revealed that both early and late apoptosis in MCF-7 and MDA-MB-231 cells were increased significantly (Fig. 2A). TUNEL staining also confirmed this result, as shown in Fig. 2B and 2C.

Furthermore, anlotinib upregulated the mRNA levels of proapoptotic proteins, including Bax, Bad, Bid, Caspase1, Caspase3, Caspase8 and Caspase9, and the protein levels of Bak, Cytochrome C, cleaved Caspase1, cleaved Caspase3, cleaved PARP1 and cleaved Caspase9 in the two cell lines. Moreover, the mRNA and protein levels of the anti-apoptotic protein Bcl-2 were downregulated after the same treatment, indicating that anlotinib promotes apoptosis and inhibits anti-apoptotic proteins in MCF-7 and MDA-MB231 cells (Fig. 2D, E and F, ${ }^{*} p<0.05,{ }^{* *} p<0.01, n=3$ ).

\subsection{Anlotinib promoted autophagy in human BC MCF-7 and MDA-MB-231 cells}

To detect whether anlotinib treatment induces autophagy in BC cells, we detected autophagy-related markers in MCF-7 and MDA-MB-231 cells with or without anlotinib treatment. We found that anlotinib induced the upregulation of LC3B and BECN1 mRNA levels (Fig. 3A and B), increased in the ratio of LC3BII to LC3BI and the protein level of BECN1, and decreased p62, as detected by western blotting (Fig. 3C, D and E) and ICC (Fig. 3A and B) in both cell lines, indicating that anlotinib induced autophagy in MCF-7 and MDA-MB-231 cells ( $\left.{ }^{\star} p<0.05,{ }^{* *} \mathrm{p}<0.01, \mathrm{n}=3\right)$. 


\subsection{Anlotinib induced apoptosis by promoting autophagy in MCF-7 and MDA-MB-231 cells}

Autophagy is a double-edged sword in tumour progression and therapy; thus, we investigated the correlation between cell apoptosis and autophagy in this study. To confirm the role of autophagy in anlotinib-induced apoptosis, MCF-7 and MDA-MB-231 cells were pre-treated with the autophagy inhibitors wortmannin (wort) and 3-methyladenine (3-MA) before anlotinib treatment. The levels of the apoptotic markers cleaved PARP1 and Bak in the anlotinib group were elevated compared with those of the DMSO group; however, these effects were reversed in the anlotinib + wort and anlotinib + 3-MA groups in both cell lines (Fig. 4). These data suggest that anlotinib at least partly, if not entirely, induces apoptosis by promoting autophagy in MCF-7 and MDA-MB-231 cells. $\left({ }^{*} \mathrm{p}<0.05,{ }^{* *} \mathrm{p}<0.01\right.$ vs DMSO, ${ }^{\#} \mathrm{p}<0.05,{ }^{\# \#} \mathrm{p}<0.01$ vs Anlotinib. $n=3$ ).

\subsection{Increased VEGFA expression was related to BC}

Anlotinib exert its function by blocking the activation of VEGFR. VEGFA is the primary and most important component of the VEGF family and is usually referred to simply as VEGF. We assessed VEGFA expression levels in $B C$ and normal tissue, as well as the expression profile in different subtypes of $B C$ in the public database UALCAN. The data indicated that VEGFA was expressed at higher levels in BC compared with that of normal tissue (Supplemental Fig. 1A); notably, VEGFA was expressed th the highest level in HER2positive BC (Supplemental Fig. 1B).

\subsection{Anlotinib exerted its function by regulating the Akt/GSK-3a pathway}

Given that anlotinib showed significant therapeutic efficacy in BC cells, we further determined the underlying mechanism. VEGFR, a target receptor of anlotinib, is an upstream molecule of the Akt signalling pathway. Hence, western blotting was used to detect Akt and GSK-3a alteration. Anlotinib decreased the phosphorylation of Akt and GSK-3a but had no effect on the protein levels of total Akt or GSK-3a in MCF-7 and MDA-MB-231 cells (Fig. 5). However, with wort and 3-MA treatment, Akt and GSK-3a phosphorylation in the anlotinib group was like the levels in the DMSO group (Fig. 5). These findings indicated that anlotinib induced apoptosis by suppressing Akt activation and enhancing GSK-3a activation and that an autophagy inhibitor reversed this alteration in MCF-7 and MDA-MB-231 cells. ( ${ }^{*} \mathrm{p}<$ $0.05,{ }^{* *} p<0.01$ vs DMSO, ${ }^{*} p<0.05,{ }^{\# \#} p<0.01$ vs Anlotinib, $n=3$ ).

\subsection{Anlotinib suppressed BC growth in vivo}

To examine the therapeutic significance in vivo, mice were subcutaneously injected with AT-3 cells to generate xenograft tumours followed by continuous 3-week intragastric treatment with anlotinib. The anlotinib group mice showed significantly decreased tumour weights and tumour volumes compared to those of the DMSO group (Fig. 6A, B and C). Consistent with the cell-based assay, the mRNA levels of 
LC3B, ATG4B and ATG5 in the anlotinib group were upregulated, and the ratio of LC3BII to LC3BI protein also increased, suggesting that anlotinib-induced autophagy also occurred in the BC xenograft mouse model. The proapoptotic protein cleaved caspase-3 was expressed at higher levels in the anlotinib group than in the DMSO group, and the anti-apoptotic protein Bcl-2 showed the opposite result. These results indicated that induction of apoptosis occurred in anlotinib-treated xenografts. Anlotinib group mice had fewer Ki67-positive cells and expressed less Cyclin D1 protein compared to those of DMSO group mice, indicating that the number of proliferating cells was reduced and the cell cycle was arrested (Fig. $6,{ }^{*} \mathrm{p}<$ $0.05,{ }^{* *} \mathrm{p}<0.01, \mathrm{DMSO}=6$, anlotinib $=8$ ). These data demonstrated that anlotinib inhibited BC growth in vivo.

\section{Discussion}

Anlotinib, an inhibitor of multiple tyrosine kinase receptors, inhibits tumour progression by inhibiting angiogenesis ${ }^{22}$, but there is no published literature on the inhibitory effect of anlotinib on BC.

Many studies have reported that anlotinib exerts antitumour effects to inhibit cell viability and proliferation in hepatocellular carcinoma (HCC), lung cancer, thyroid cancer and osteosarcoma ${ }^{23-26}$. Our study found that anlotinib also inhibited the cell viability and proliferation of BC cells, which was consistent with the effects in other tumours. Cell proliferation is regulated by the cell cycle, and Cyclin is an important regulatory molecule that drives the cell cycle. Cyclin D1 is a member of the cyclin family that is activated in the $\mathrm{G} 1$ phase and drives $\mathrm{G} 1 / \mathrm{S}$ phase transitions. Cyclin D1 binds to cyclin-dependent kinase 4 and cyclin-dependent kinase 6, induces excessive retinoblastoma protein phosphorylation, thereby shortening the $\mathrm{G} 1$ phase, promoting excessive cell proliferation and leading to tumourigenesis ${ }^{27}$. This study found that the expression of Cyclin D1 mRNA and protein in BC cells decreased significantly after anlotinib treatment. In contrast, the G1 phase decreased unexpectedly. It is possible that the increased G2/M phase and specific and deeper mechanisms require further investigation. The above evidence suggests that anlotinib inhibits the proliferation of BC cells by prolonging the cell cycle. Similar to our results, anlotinib in thyroid cancer causes abnormal spindle assembly and G2/M arrest, inhibiting cell cycle progression ${ }^{28}$.

Autophagy is an important cellular mechanism that plays a "housekeeping" role in normal physiological processes, including the removal of longevity, aggregation and misfolded proteins, removal of damaged organelles, and the regulation of growth and ageing. In tumour cells, autophagy is usually activated during anticancer treatments such as radiation therapy, chemotherapy, and targeted therapy. This may be a cytoprotective mechanism that also causes excessive autophagy in the cell, namely, excessive selfdigestion, and induces phagocytic cell death, which is also known as type II programmed cell death ${ }^{29}$. A study found that anlotinib induced autophagy in human lung cancer cells in a time- and concentrationdependent manner and increased the ratio of LC3BII/I protein and the protein expression level of BECN1. Using the autophagy inhibitors 3-MA and BECN1, small interfering RNA reversed the autophagy effect induced by anlotinib; unexpectedly, it enhanced the inhibitory effect of anlotinib on cell proliferation, 
making the anticancer effect of anlotinib more sensitive and strengthening its inhibition of angiogenesis 25 . This suggests that the induction of autophagy in human lung cancer cells by anlotinib is a cytoprotective effect. In our study, anlotinib also induced autophagy in MCF-7 and MDA-MB-231 human $B C$ cells. These results showed significantly increased mRNA expression levels of LC3B and BECN1 and the ratio of LC3BII/I protein and BECN1 protein levels and decreased P62 protein levels, suggesting that promoting autophagic cell death may be an important mechanism by which anlotinib inhibits BC cell growth.

Apoptosis is a common programmed cell death and plays a key role in the development of diseases, including cancer. Cancer cells evade apoptosis, thereby achieving excessive proliferation and surviving under hypoxic conditions and with drug resistance ${ }^{30}$; thus, promoting tumour cell apoptosis has become an important strategy for the treatment of cancer. Studies have shown that anlotinib exerts its antitumour effects on HCC, thyroid cancer, osteosarcoma and lung cancer by promoting apoptosis ${ }^{23-26,31}$. Anlotinib significantly inhibited colony formation and promoted apoptosis in HCC and thyroid cancer in vitro ${ }^{23,24}$. It upregulated the pro-apoptotic molecule Bax and inhibited the anti-apoptotic proteins Bcl-2 and Survivin to kill tumour cells. In addition, animal experiments demonstrated that anlotinib reduced the volumes and weights of transplanted tumours ${ }^{24}$. In thyroid cancer, anlotinib caused abnormal spindle assembly and G2/M arrest, promoted the activation of cleaved-Caspase 3 and cleaved PARP, and activated TP53 ${ }^{23}$. Similar to the above experimental results, we found that anlotinib increased the mRNA and protein levels of proapoptotic proteins and inhibited the mRNA and protein levels of the anti-apoptotic protein Bcl-2 in MCF-7 and MDA-MB-231 BC cells, thereby exerting an antitumour effect.

Autophagy and apoptosis often occur in the same cells with the same upstream cellular signals activated by the endoplasmic reticulum, such as extracellular regulated protein kinases (ERK)/ activating transcription factor 4 (ATF4), Inositol-requiring enzyme-1a, ATF6, and $\mathrm{Ca}^{2+}$. On the one hand, autophagy not only blocks the induction of apoptosis by inhibiting the activation of apoptosis-associated caspases and reduces cell damage but also induces apoptosis. On the other hand, activation of apoptosis-related proteins also suppresses autophagy by degrading autophagy-related proteins such as BECN1, autophagy-related protein 4D (ATG4D), ATG3 and ATG5, but the specific mechanisms of their mutual regulation in $B C$ cells need further study ${ }^{32}$. However, in our study, we utilized the autophagy inhibitors wort and 3-MA before anlotinib treatment and found that inhibition of autophagy reversed anlotinibinduced apoptosis in BC cells. We obtained the opposite results compared with that reported in lung cancer cells ${ }^{25}$. This is probably due to the different concentrations of autophagy inhibitors and different cells, but it remains unclear and needs further study to clarify the crosstalk between apoptosis and autophagy.

Evidence indicates that Akt is a key molecule in both autophagy and apoptosis because it is the upstream signal of mammalian target of rapamycin complex and $\mathrm{JNK}^{33-35}$. It is also a downstream protein of VEGFR signalling. Previous studies reported that anlotinib inhibits Erk and Akt signal transduction pathways to regulate cell growth in $\mathrm{HCC}$ cells ${ }^{24}$. Hence, we investigated Akt signalling and found that 
inactivated Akt/GSK-3a signalling in anlotinib-induced apoptosis was reversed by autophagy inhibitors, suggesting that anlotinib-induced autophagy promotes apoptosis by impacting Akt/GSK-3a signalling.

In summary, our study demonstrated that anlotinib inhibited the growth of $\mathrm{BC}$ cells via promoting apoptosis through autophagy mediated by Akt/GSK-3a signalling and may be an effective new drug for $\mathrm{BC}$ treatment.

\section{Abbreviations}

\begin{tabular}{|ll|}
\hline 3-methyladenine & 3-MA \\
\hline activating transcription factor 4 & ATF4 \\
\hline autophagy-related protein & ATG \\
\hline Breast cancer & BC \\
\hline extracellular regulated protein kinases & ERK \\
\hline fibroblast growth factor receptor & FGFR \\
\hline paraformaldehyde & PFA \\
\hline platelet-derived growth factor receptor & PDGFR \\
\hline vascular endothelial growth factor receptor & VEGFR \\
\hline wortmannin & wort \\
\hline
\end{tabular}

\section{Declarations}

\section{Ethical Approval and Consent to participate}

All animal experiments were approved by the Ethics Committee (Approval number: 20190703).

\section{Consent for publication}

Written informed consent for publication was obtained from all participants.

\section{Availability of supporting data}

Supporting data were from the public database UALCAN ( http://ualcan.path.uab.edu/index.html ).

\section{Competing interests}

The authors disclose no potential conflicts of interest.

\section{Funding}


Medical Science Research Foundation from Beijing Medical and Health Foundation (F2190E), and Medical Science Research Foundation from Bethune Charitable Foundation (B19358ET).

\section{Authors' contributions}

SC: study concept and design, acquisition of data, analysis and interpretation of data, drafting of the manuscript, statistical analysis; PZ: acquisition of data; XW: acquisition of data; YJ: acquisition of data; XZ: statistical analysis; PZ: study concept and design, revising of the manuscript; HY: study concept and design.

\section{Acknowledgements}

We thank the financial support from Beijing Medical and Health Foundation and Bethune Charitable Foundation and writing assistance provided by Spring Nature Author Services.

\section{References}

[1] Siegel RL, Miller KD, Jemal A. Cancer statistics, 2019. CA: A Cancer Journal for Clinicians. 2019; 69: 734.

[2] Chen W, Zheng R, Baade PD, et al. Cancer statistics in China, 2015. CA: A Cancer Journal for Clinicians. 2016; 66: 115-32.

[3] Li L, Gao Q, Xu G, et al. Postoperative recurrence analysis of breast cancer patients based on clinical serum markers using discriminant methods. CANCER BIOMARK. 2017; 19: 403-9.

[4] Ma R, Feng Y, Lin S, et al. Mechanisms involved in breast cancer liver metastasis. J TRANSL MED. $2015 ; 13$.

[5] Zhong C, Chen F, Yang J, et al. Pharmacokinetics and disposition of anlotinib, an oral tyrosine kinase inhibitor, in experimental animal species. ACTA PHARMACOL SIN. 2018; 39: 1048-63.

[6] Lin B, Song X, Yang D, Bai D, Yao Y, Lu N. Anlotinib inhibits angiogenesis via suppressing the activation of VEGFR2, PDGFRß and FGFR1. GENE. 2018; 654: 77-86.

[7] Mross K, Frost A, Steinbild S, et al. A Phase I Dose-Escalation Study of Regorafenib (BAY 73-4506), an Inhibitor of Oncogenic, Angiogenic, and Stromal Kinases, in Patients with Advanced Solid Tumors. CLIN CANCER RES. 2012; 18: 2658-67.

[8] Strumberg D, Richly H, Hilger RA, et al. Phase I Clinical and Pharmacokinetic Study of the Novel Raf Kinase and Vascular Endothelial Growth Factor Receptor Inhibitor BAY 43-9006 in Patients With Advanced Refractory Solid Tumors. J CLIN ONCOL. 2005; 23: 965-72. 
[9] Huang L, Huang Z, Bai Z, Xie R, Sun L, Lin K. Development and strategies of VEGFR-2/KDR inhibitors. FUTURE MED CHEM. 2012; 4: 1839-52.

[10] Wesche J, Haglund K, Haugsten EM. Fibroblast growth factors and their receptors in cancer. BIOCHEM J. 2011; 437: 199-213.

[11] Turner N, Grose R. Fibroblast growth factor signalling: from development to cancer. NAT REV CANCER. 2010; 10: 116-29.

[12] Knights V, Cook SJ. De-regulated FGF receptors as therapeutic targets in cancer. Pharmacol Ther. 2010; 125: 105-17.

[13] Fibroblast growth factors and their receptors in cancer.

[14] Knights V, Cook SJ. De-regulated FGF receptors as therapeutic targets in cancer. PHARMACOL THERAPEUT. 2010; 125: 105-17.

[15] Turner N, Grose R. Fibroblast growth factor signalling: from development to cancer. NAT REV CANCER. 2010; 10: 116-29.

[16] Kakiuchi-Kiyota S, Lappin PB, Heintz C, et al. Expression of proto-oncogene cFMS protein in lung, breast, and ovarian cancers. Appl Immunohistochem Mol Morphol. 2014; 22: 188-99.

[17] Ashton S, Song YH, Nolan J, et al. Aurora kinase inhibitor nanoparticles target tumors with favorable therapeutic index in vivo. SCI TRANSL MED. 2016; 8: 317r-325r.

[18] Wang C, Chen J, Cao W, Sun L, Sun H, Liu Y. Aurora-B and HDAC synergistically regulate survival and proliferation of lymphoma cell via AKT, mTOR and Notch pathways. EUR J PHARMACOL. 2016; 779: 1-7.

[19] Ambrogio C, Gómez-López G, Falcone M, et al. Combined inhibition of DDR1 and Notch signaling is a therapeutic strategy for KRAS-driven lung adenocarcinoma. NAT MED. 2016; 22: 270-7.

[20] Xie C, Wan X, Quan H, et al. Preclinical characterization of anlotinib, a highly potent and selective vascular endothelial growth factor receptor-2 inhibitor. CANCER SCI. 2018; 109: 1207-19.

[21] Beavis PA, Divisekera U, Paget C, et al. Blockade of A2A receptors potently suppresses the metastasis of CD73+ tumors. Proceedings of the National Academy of Sciences. 2013; 110: 14711-6.

[22] Han B, Li K, Zhao Y, et al. Anlotinib as a third-line therapy in patients with refractory advanced nonsmall-cell lung cancer: a multicentre, randomised phase II trial (ALTER0302). BRIT J CANCER. 2018; 118: 654-61.

[23] Ruan X, Shi X, Dong Q, et al. Antitumor effects of anlotinib in thyroid cancer. ENDOCR-RELAT CANCER. 2019: 153-64. 
[24] He C, Wu T, Hao Y. Anlotinib induces hepatocellular carcinoma apoptosis and inhibits proliferation via Erk and Akt pathway. BIOCHEM BIOPH RES CO. 2018; 503: 3093-9.

[25] Liang L, Hui K, Hu C, et al. Autophagy inhibition potentiates the anti-angiogenic property of multikinase inhibitor anlotinib through JAK2/STAT3/VEGFA signaling in non-small cell lung cancer cells. J EXP CLIN CANC RES. 2019; 38.

[26] Wang G, Sun M, Jiang Y, et al. Anlotinib, a novel small molecular tyrosine kinase inhibitor, suppresses growth and metastasisvia dual blockade of VEGFR2 and MET in osteosarcoma. INT J CANCER. 2019; 145: 979-93.

[27] Ahlin C, Lundgren C, Embretsén-Varro E, Jirström K, Blomqvist C, Fjälllskog ML. High expression of cyclin D1 is associated to high proliferation rate and increased risk of mortality in women with ERpositive but not in ER-negative breast cancers. BREAST CANCER RES TR. 2017; 164: 667-78.

[28] Ruan X, Shi X, Dong Q, et al. Antitumor effects of anlotinib in thyroid cancer. ENDOCR-RELAT CANCER. 2019: 153-64.

[29] Ravanan P, Srikumar IF, Talwar P. Autophagy: The spotlight for cellular stress responses. LIFE SCI. 2017; 188: 53-67.

[30] Matsuura K, Canfield K, Feng W, Kurokawa M. Metabolic Regulation of Apoptosis in Cancer. INT REV CEL MOL BIO. 2016; 327: 43-87.

[31] Sun Y, Du F, Gao M, et al. Anlotinib for the Treatment of Patients with Locally Advanced or Metastatic Medullary Thyroid Cancer. THYROID. 2018; 28: 1455-61.

[32] Song S, Tan J, Miao Y, Li M, Zhang Q. Crosstalk of autophagy and apoptosis: Involvement of the dual role of autophagy under ER stress. J CELL PHYSIOL. 2017; 232: 2977-84.

[33] Heras-Sandoval D, Pérez-Rojas JM, Hernández-Damián J, Pedraza-Chaverri J. The role of $\mathrm{PI} 3 \mathrm{~K} / \mathrm{AKT} / \mathrm{mTOR}$ pathway in the modulation of autophagy and the clearance of protein aggregates in neurodegeneration. CELL SIGNAL. 2014; 26: 2694-701.

[34] Zhang W, Hou J, Yan X, et al. Platycodon grandiflorum Saponins Ameliorate Cisplatin-Induced Acute Nephrotoxicity through the NF-KB-Mediated Inflammation and PI3K/Akt/Apoptosis Signaling Pathways. NUTRIENTS. 2018; 10: 1328.

[35] Yu Y, Lv F, Liang D, et al. HOTAIR may regulate proliferation, apoptosis, migration and invasion of MCF-7 cells through regulating the P53/Akt/JNK signaling pathway. BIOMED PHARMACOTHER. 2017; 90: 555-61.

\section{Figures}



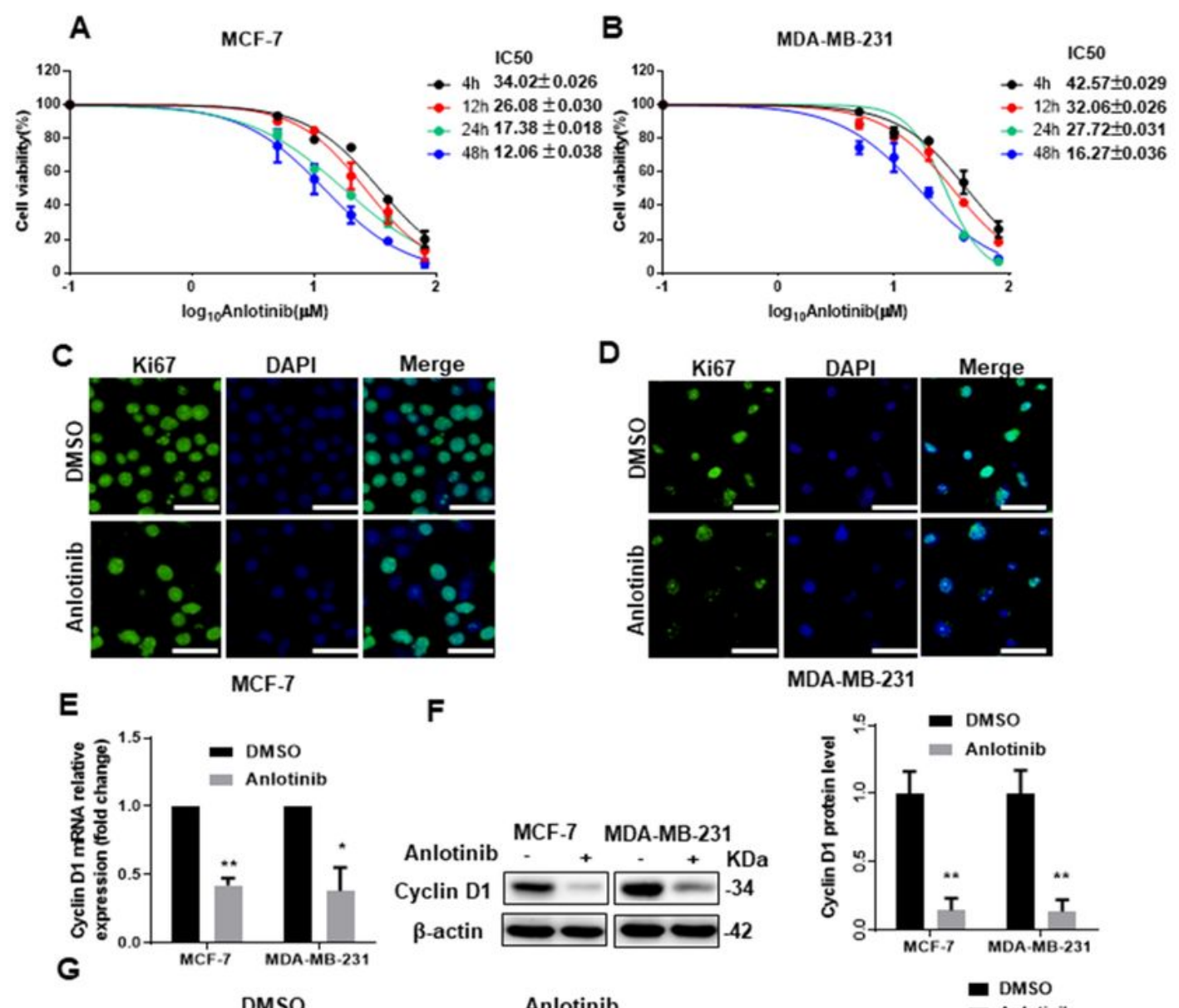

$\mathbf{F}$

D

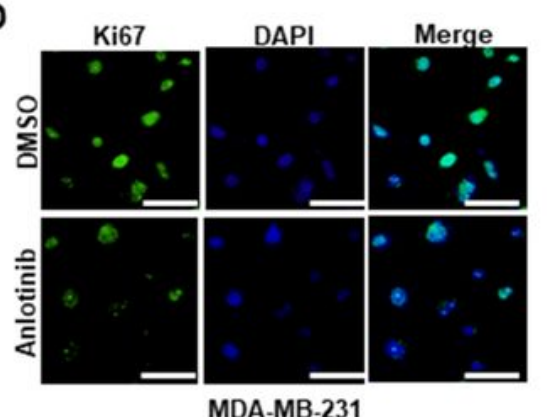

MDA-MB-231
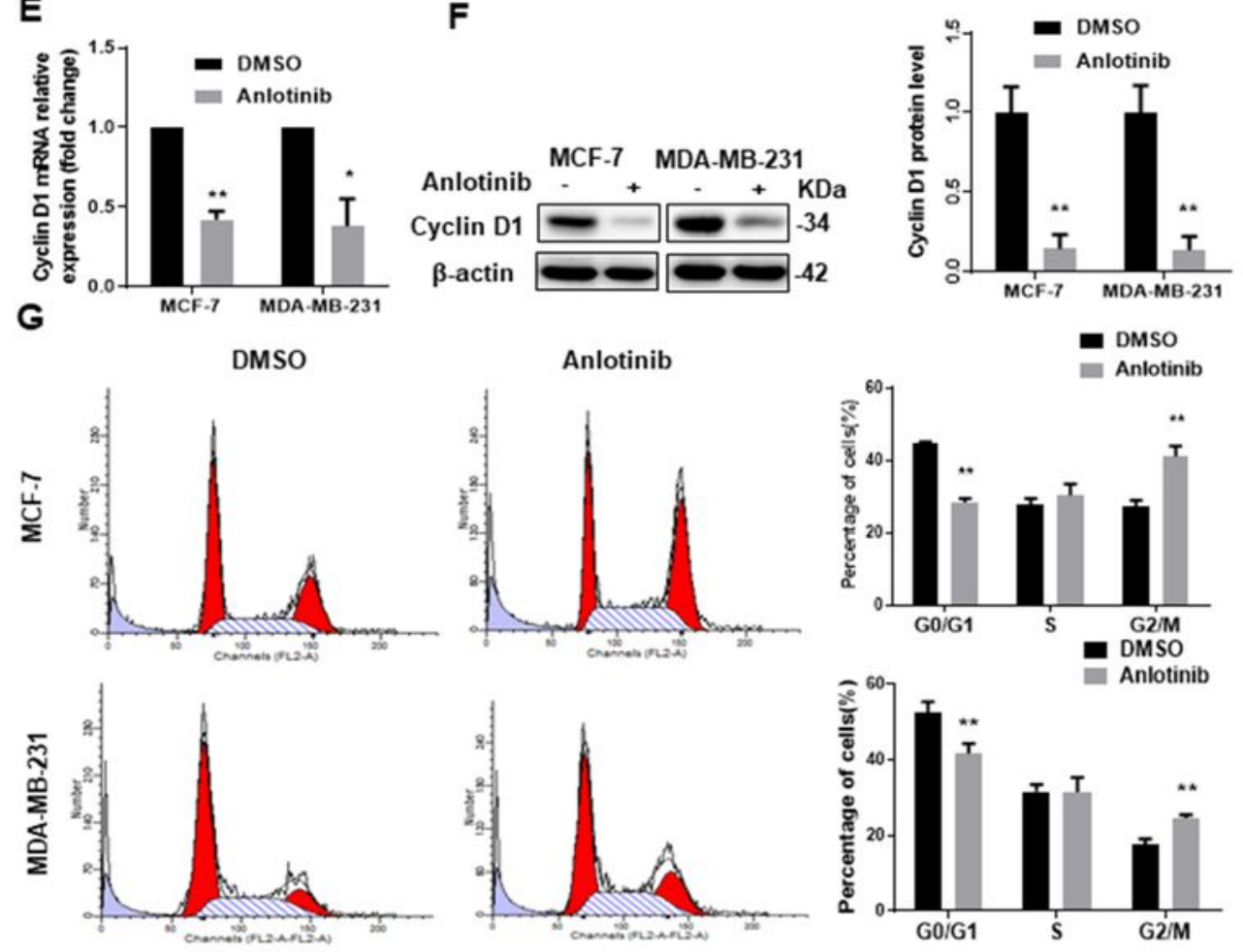

\section{Figure 1}

Anlotinib suppressed proliferation and regulated the cell cycle of human BC cells. A and B. Dose-response curves of anlotinib treatment. Cells were cultured with anlotinib at various concentrations for 4, 12, 24 and $48 \mathrm{~h}$, and cell viability was detected by CCK-8 assay. C and D. Representative images of Ki67 staining. The MCF-7 and MDA-MB-231 cells were stained after anlotinib treatment with $10 \mu \mathrm{M}$ for $12 \mathrm{~h}$. Scale bars: $50 \mu \mathrm{m}$. E and F. The mRNA and protein levels of Cyclin D1 were significantly decreased after 
anlotinib treatment with 10 $\mu \mathrm{M}$ for $24 \mathrm{~h}$ in MCF-7 and MDA-MB-231 cells. G. Anlotinib-induced G2/M phase arrest in BC cells. MCF-7 and MDA-MB-231 cells were treated with DMSO or $10 \mu \mathrm{M}$ anlotinib for 12 $\mathrm{h}$ and stained with PI. The percentage of cells in G0/G1, S and G2/M phase were calculated and plotted. The data are shown as the mean \pm SD. ${ }^{*} \mathrm{p}<0.05,{ }^{* *} \mathrm{p}<0.01$ vs DMSO, $\mathrm{n}=3$.
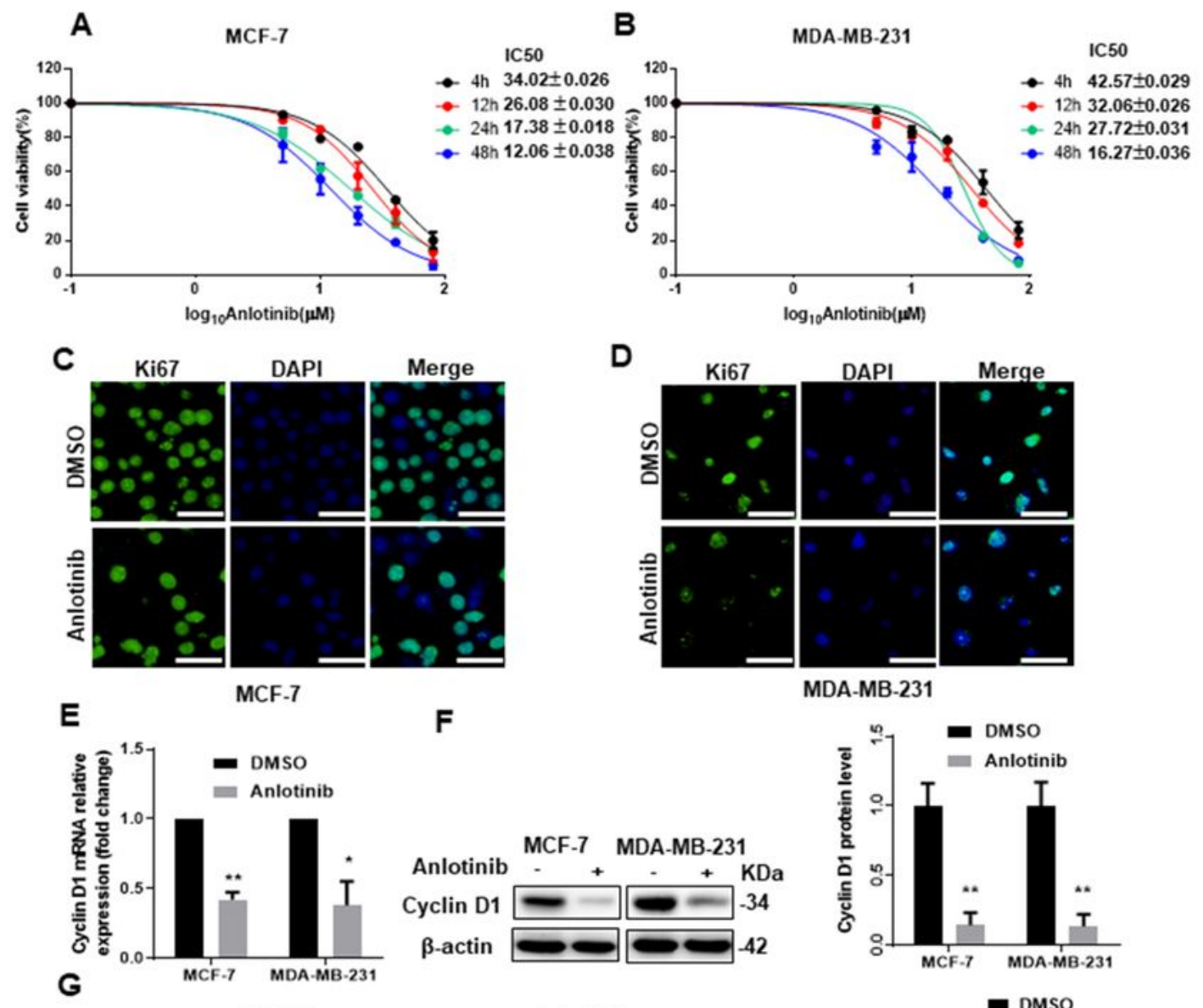

F

D

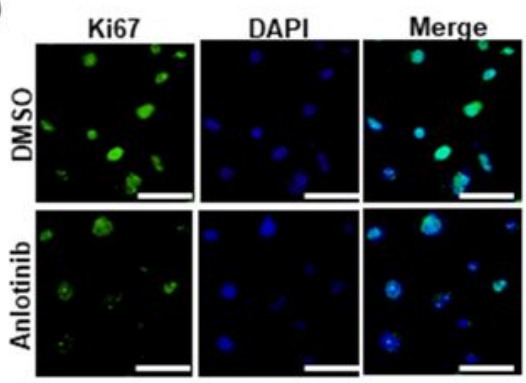

MDA-MB-231
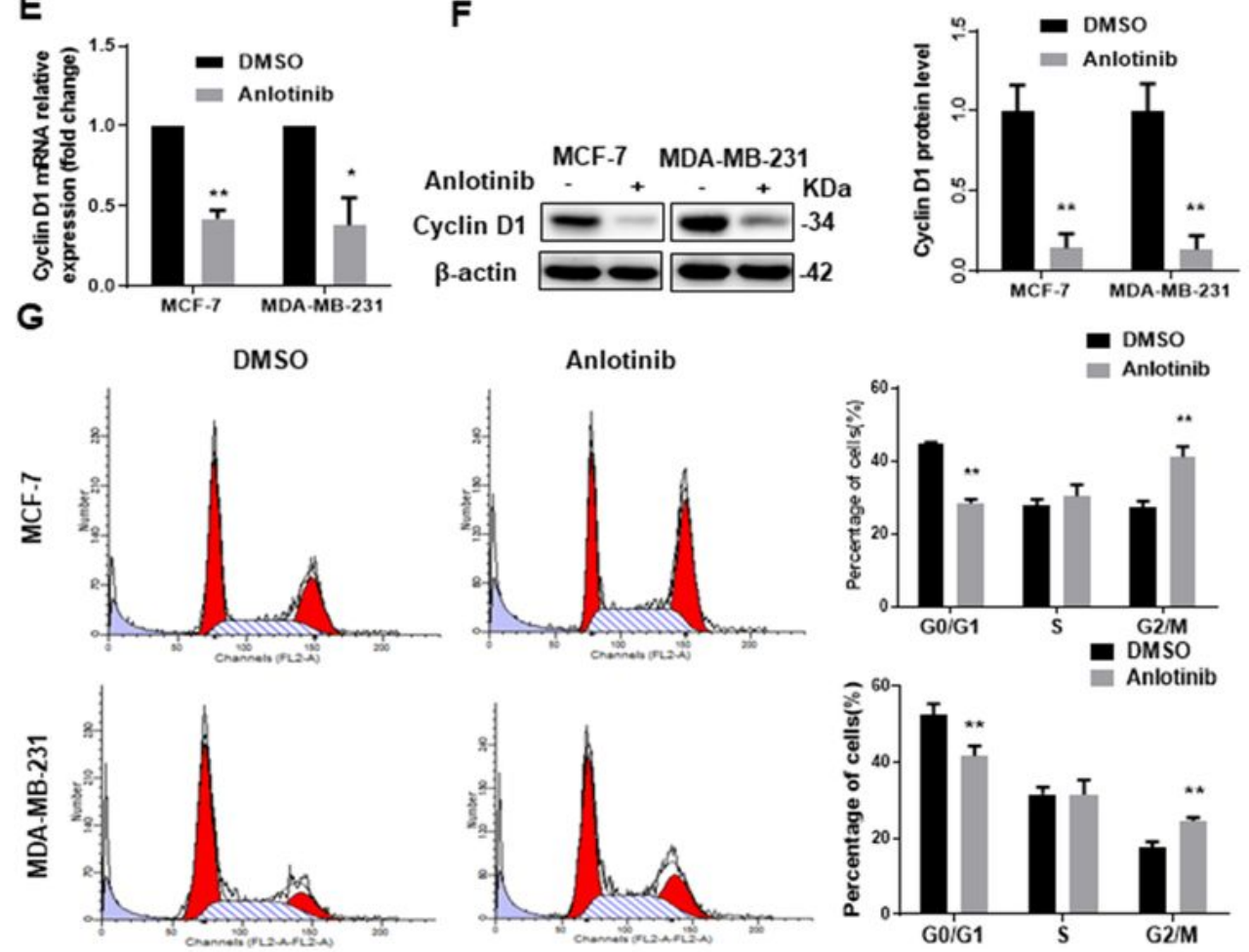

Figure 1 
Anlotinib suppressed proliferation and regulated the cell cycle of human BC cells. A and B. Dose-response curves of anlotinib treatment. Cells were cultured with anlotinib at various concentrations for $4,12,24$ and $48 \mathrm{~h}$, and cell viability was detected by CCK-8 assay. C and D. Representative images of Ki67 staining. The MCF-7 and MDA-MB-231 cells were stained after anlotinib treatment with $10 \mu \mathrm{M}$ for $12 \mathrm{~h}$. Scale bars: $50 \mu \mathrm{m}$. E and F. The mRNA and protein levels of Cyclin D1 were significantly decreased after anlotinib treatment with $10 \mu \mathrm{M}$ for $24 \mathrm{~h}$ in MCF-7 and MDA-MB-231 cells. G. Anlotinib-induced G2/M phase arrest in BC cells. MCF-7 and MDA-MB-231 cells were treated with DMSO or $10 \mu \mathrm{M}$ anlotinib for 12 $h$ and stained with PI. The percentage of cells in G0/G1, S and G2/M phase were calculated and plotted. The data are shown as the mean \pm SD. ${ }^{*} p<0.05,{ }^{\star \star} p<0.01$ vs DMSO, $n=3$. 
A
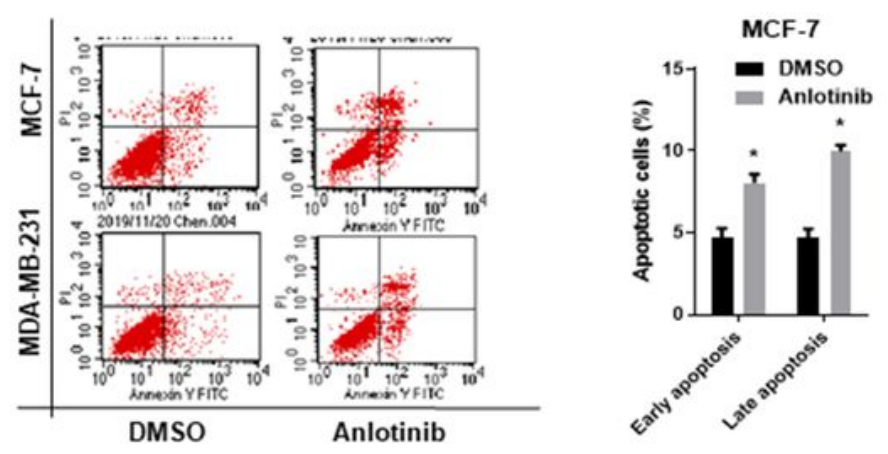

MDA-MB-231
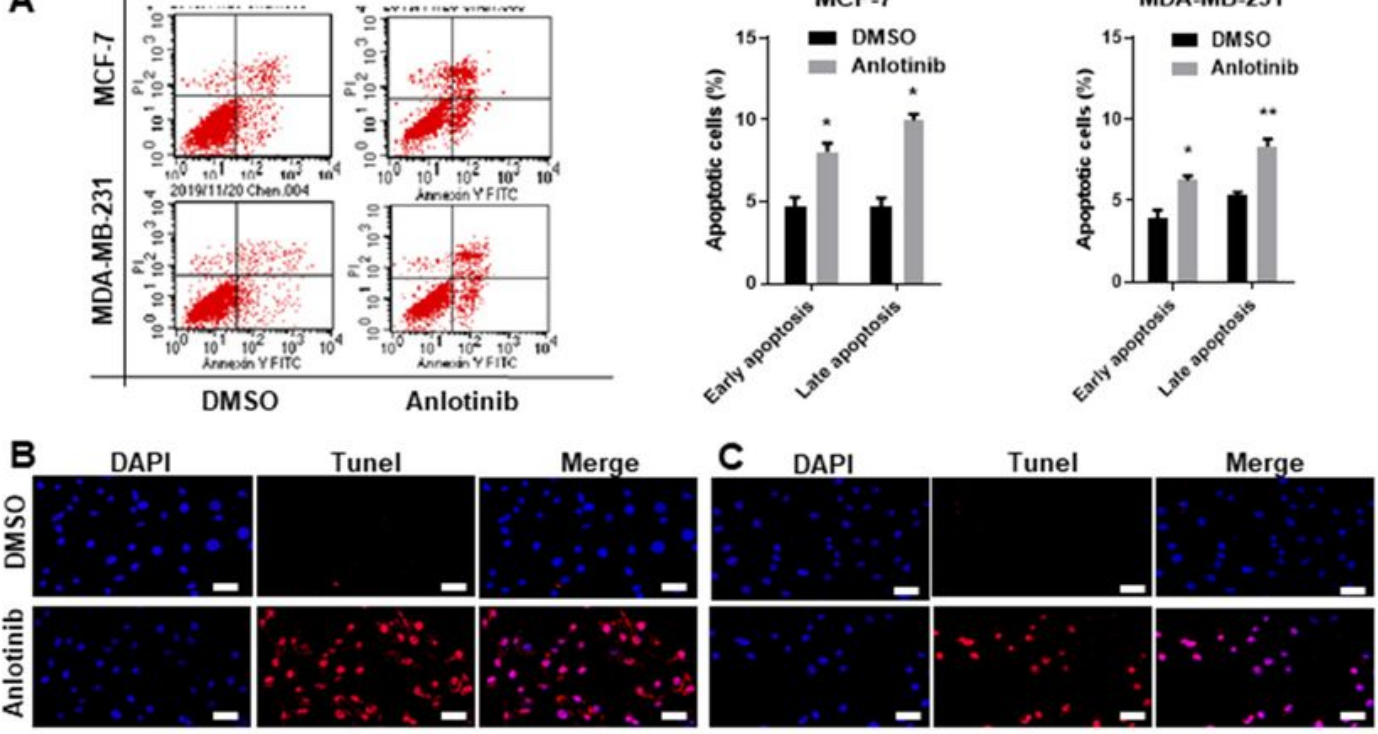

MCF-7

D

MCF-7

MDA-MB-231

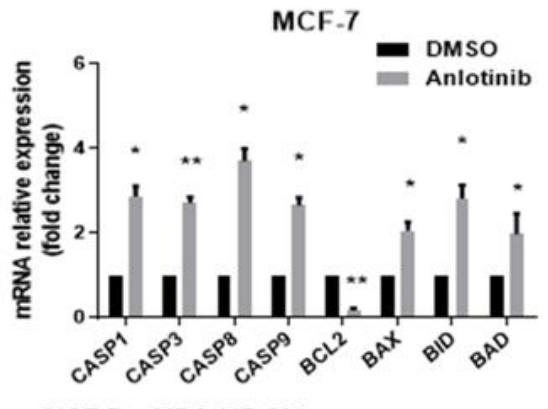

E

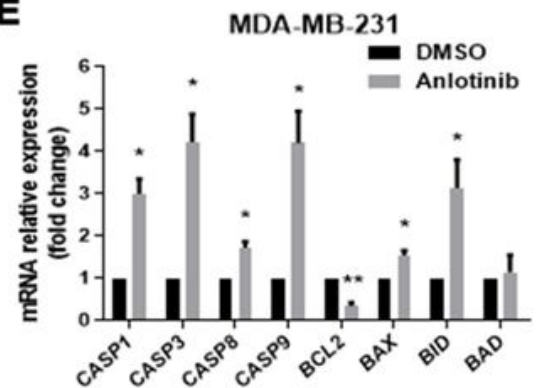

F

MCF-7 MDA-MB-231
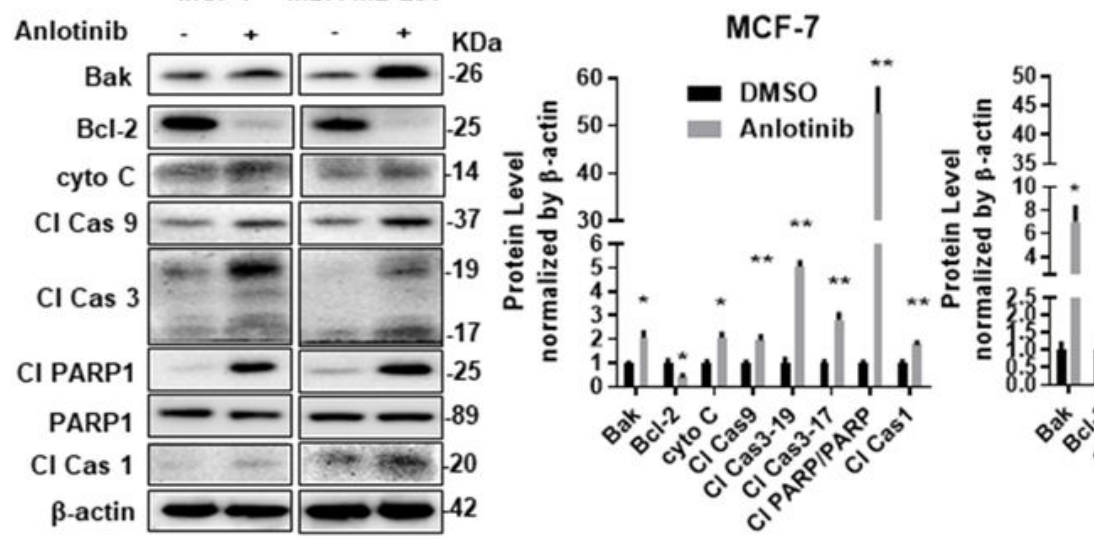

MDA-MB-231

- DMSO

- Anlotinib |
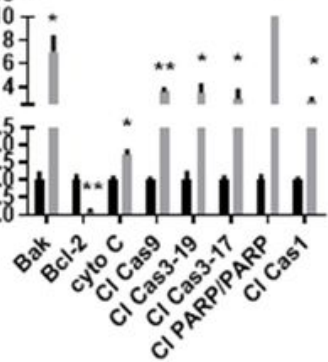

\section{Figure 2}

Anlotinib promoted apoptosis in human BC cells. The MCF-7 and MDA-MB-231 cells were induced by anlotinib with $10 \mu \mathrm{M}$ for $24 \mathrm{~h}$. A. The ratio of early and late apoptotic cells was measured in MCF-7 and MDA-MB-231 cells treated with DMSO or anlotinib. Apoptosis was detected by Annexin V-FITC and propidium iodide $(\mathrm{PI})$ staining. $\mathrm{B}$ and $\mathrm{C}$. The apoptotic level in the anlotinib-treated cells was detected by TUNEL staining. Scale bars: $25 \mu \mathrm{m}$. D and E. The mRNA level of apoptotic-associated molecules was 
increased markedly in anlotinib-treated cells. F. Western blot showing the expression of apoptosisassociated proteins (left). Quantitative summary of the blots in the right panel; the data were normalized to $\beta$-actin. The data are shown as the mean $\pm S D$. ${ }^{*} p<0.05$, ${ }^{*} p p<0.01$ vs DMSO, $n=3$.

A
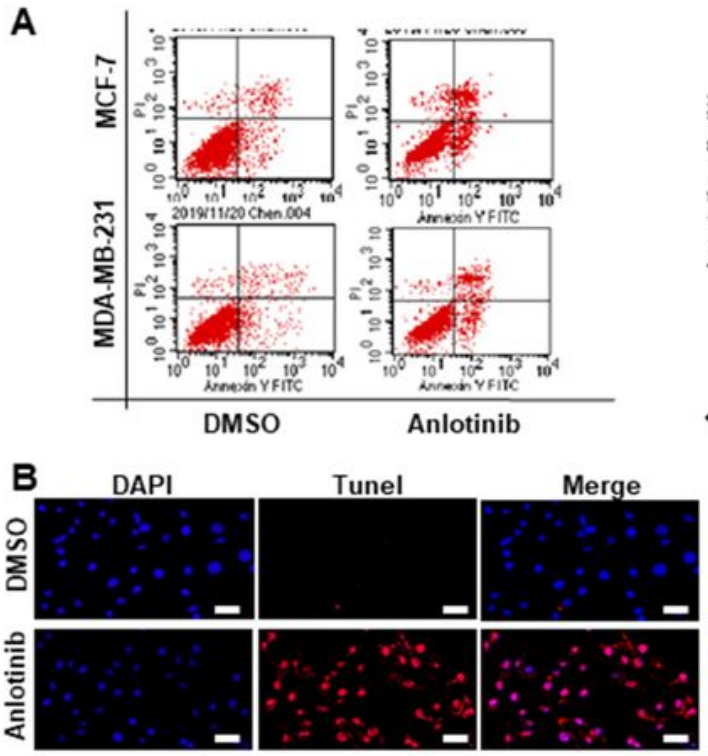

MCF-7

D

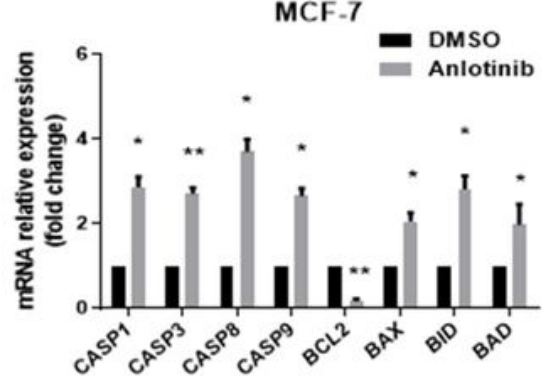

$\mathbf{F}$

MCF-7 MDA-MB-231

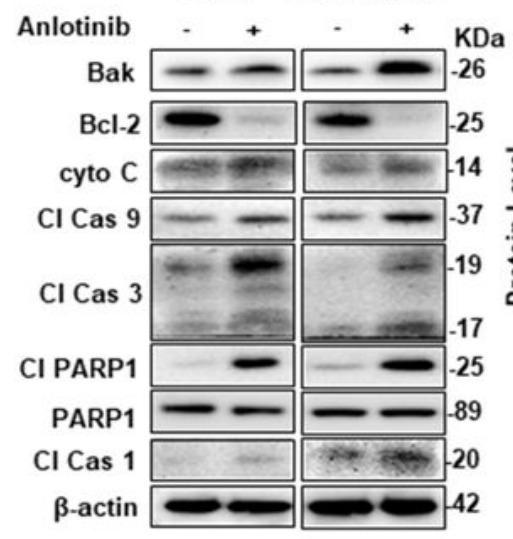

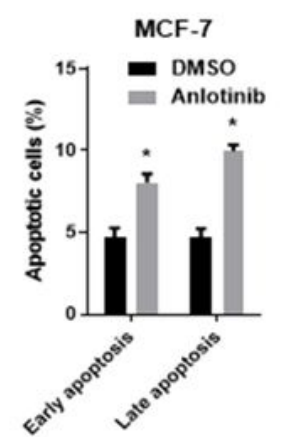
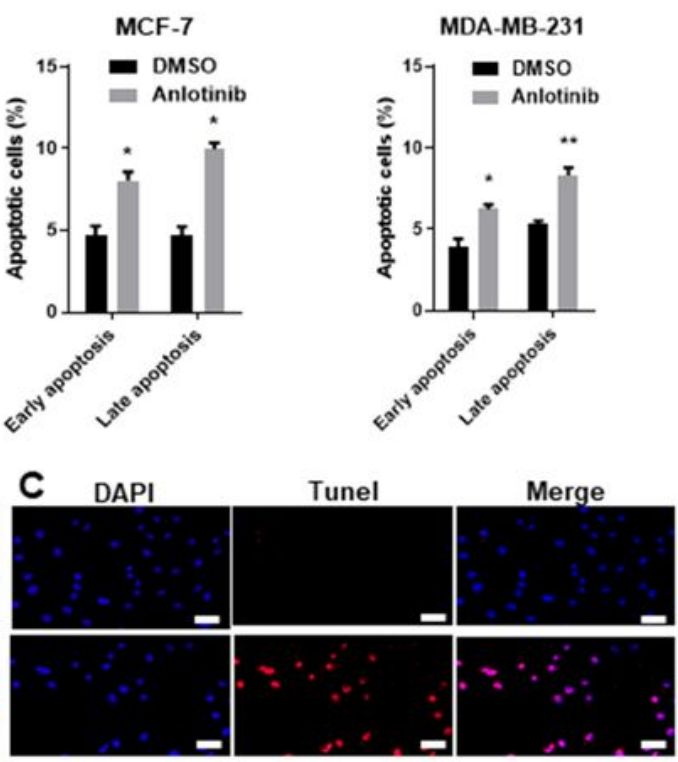

MDA-MB-231

E

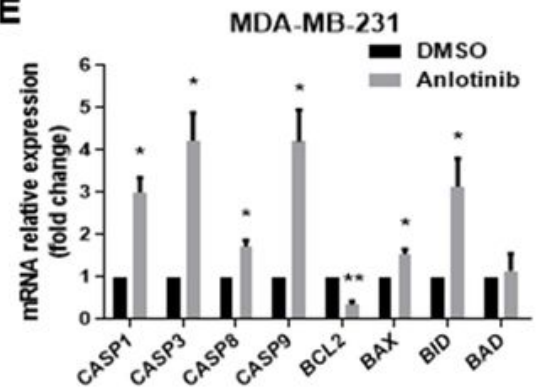

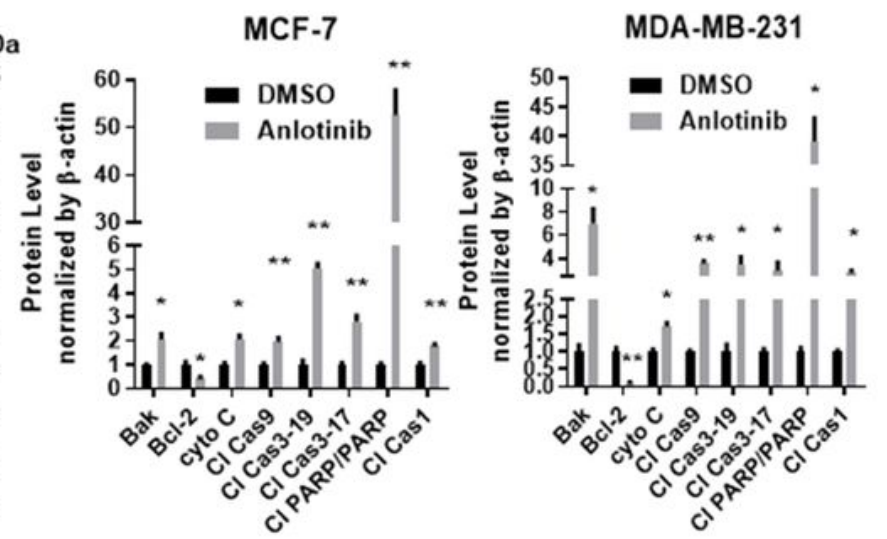

Figure 2

Anlotinib promoted apoptosis in human BC cells. The MCF-7 and MDA-MB-231 cells were induced by anlotinib with $10 \mu \mathrm{M}$ for $24 \mathrm{~h}$. A. The ratio of early and late apoptotic cells was measured in MCF-7 and 
MDA-MB-231 cells treated with DMSO or anlotinib. Apoptosis was detected by Annexin V-FITC and propidium iodide $(\mathrm{PI})$ staining. $\mathrm{B}$ and $\mathrm{C}$. The apoptotic level in the anlotinib-treated cells was detected by TUNEL staining. Scale bars: $25 \mu \mathrm{m}$. D and E. The mRNA level of apoptotic-associated molecules was increased markedly in anlotinib-treated cells. F. Western blot showing the expression of apoptosisassociated proteins (left). Quantitative summary of the blots in the right panel; the data were normalized to $\beta$-actin. The data are shown as the mean \pm SD. ${ }^{*} p<0.05$, ** $p<0.01$ vs DMSO, $n=3$.

A

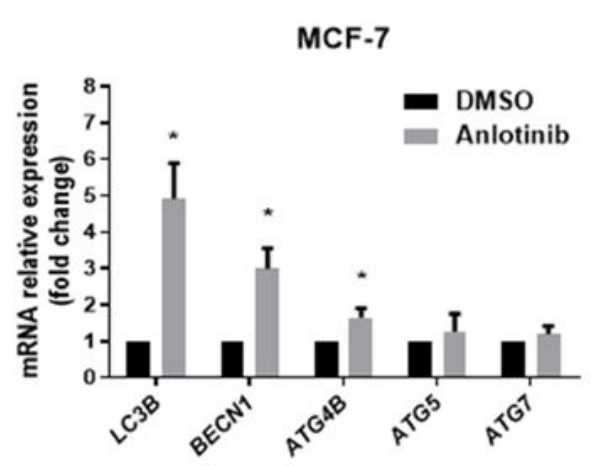

B

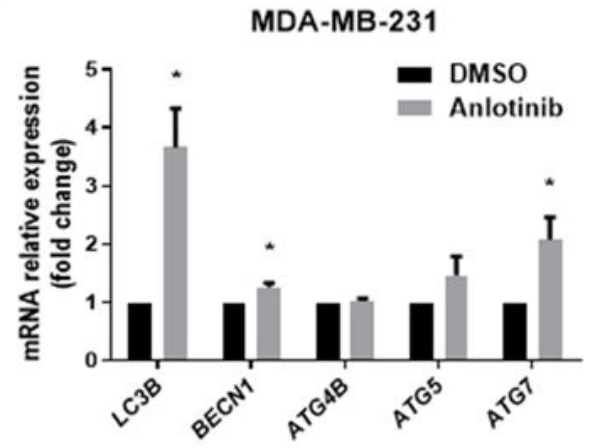

C

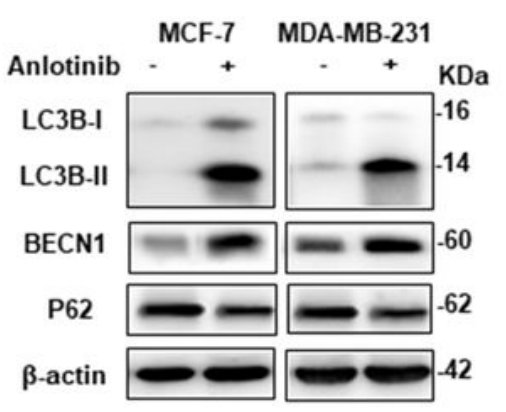

$\mathbf{F}$

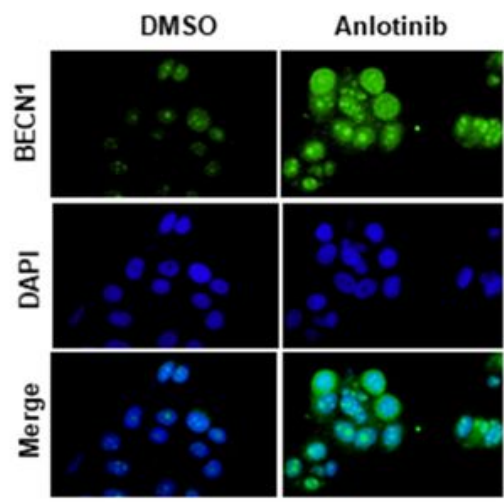

MCF-7
E

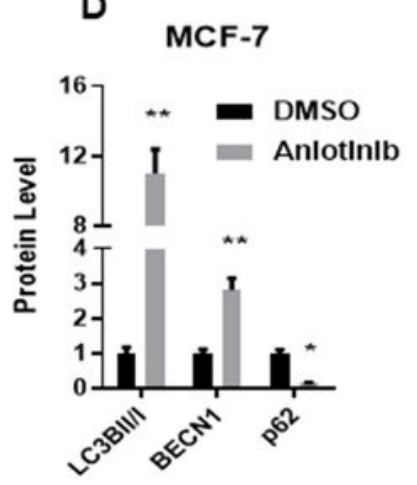

G

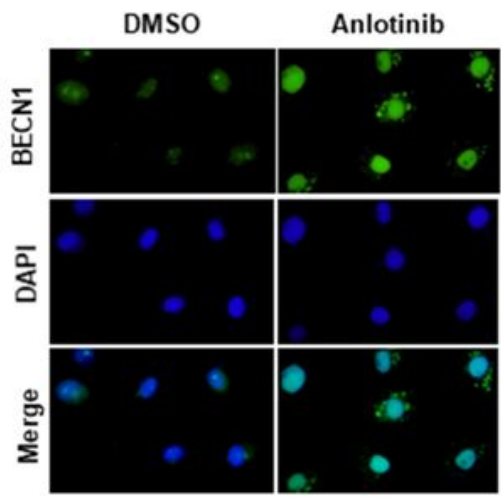

MDA-MB-231

Figure 3 
Anlotinib induced autophagy with $10 \mu \mathrm{M}$ for $24 \mathrm{~h}$ in human BC cell lines. A and B. The mRNA level of autophagy-related molecules was increased markedly in anlotinib-treated cells. C. Western blot showing the expression of autophagy-related proteins. $D$ and $E$. Quantitative summary of the blots in panel $C$ for autophagy-related proteins; the data were normalized to $\beta$-actin. $F$ and $G$. Representative images of BECN1 staining. The data are shown as the mean $\pm S D$. ${ }^{*} p<0.05$, ${ }^{*} p<0.01$ vs DMSO, $n=3$.

A

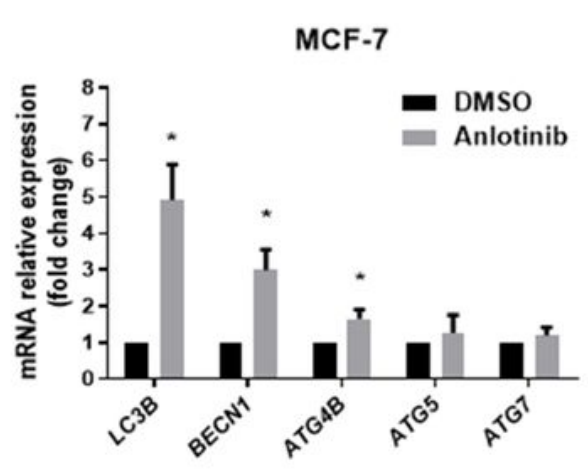

B

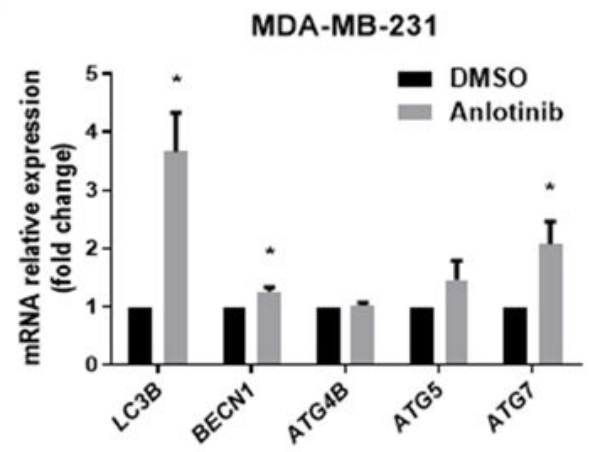

c

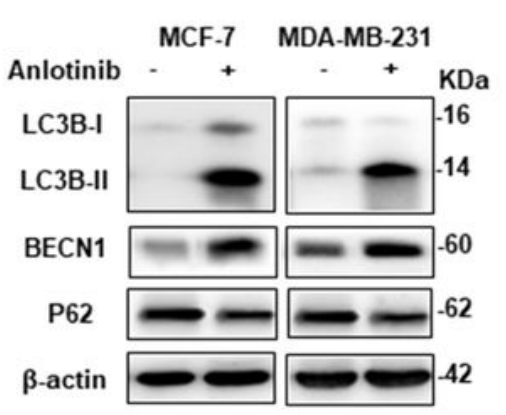

$\mathbf{F}$

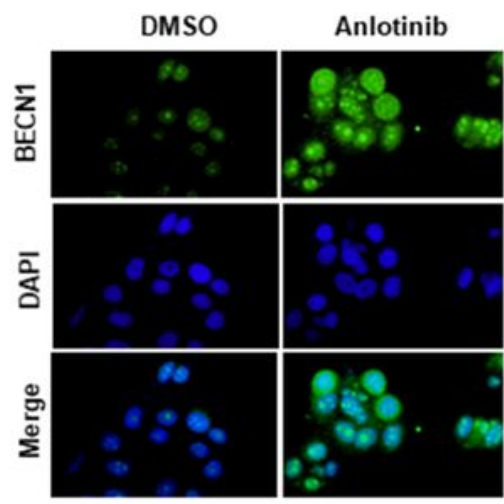

MCF-7
$\mathbf{E}$

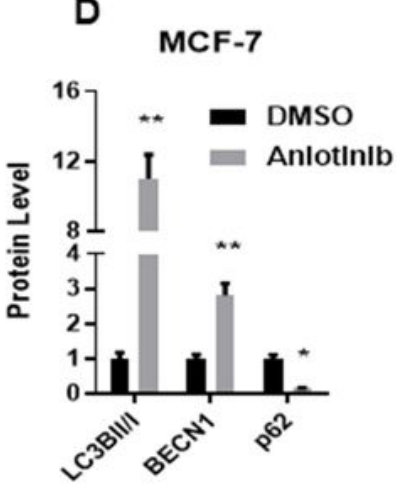

G

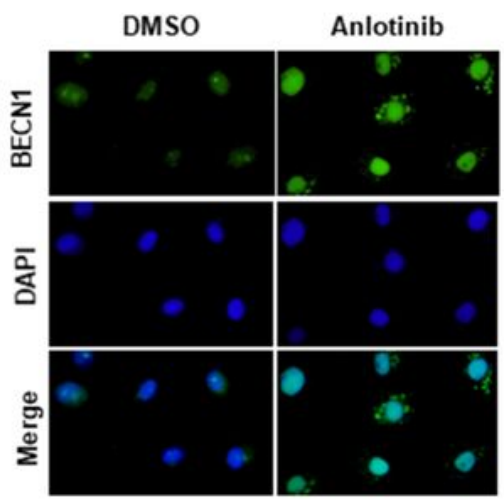

MDA-MB-231 
Anlotinib induced autophagy with $10 \mu \mathrm{M}$ for $24 \mathrm{~h}$ in human BC cell lines. A and B. The mRNA level of autophagy-related molecules was increased markedly in anlotinib-treated cells. C. Western blot showing the expression of autophagy-related proteins. $D$ and $E$. Quantitative summary of the blots in panel $C$ for autophagy-related proteins; the data were normalized to $\beta$-actin. F and G. Representative images of BECN1 staining. The data are shown as the mean \pm SD. ${ }^{*} p<0.05,{ }^{*} p<0.01$ vs DMSO, $n=3$.

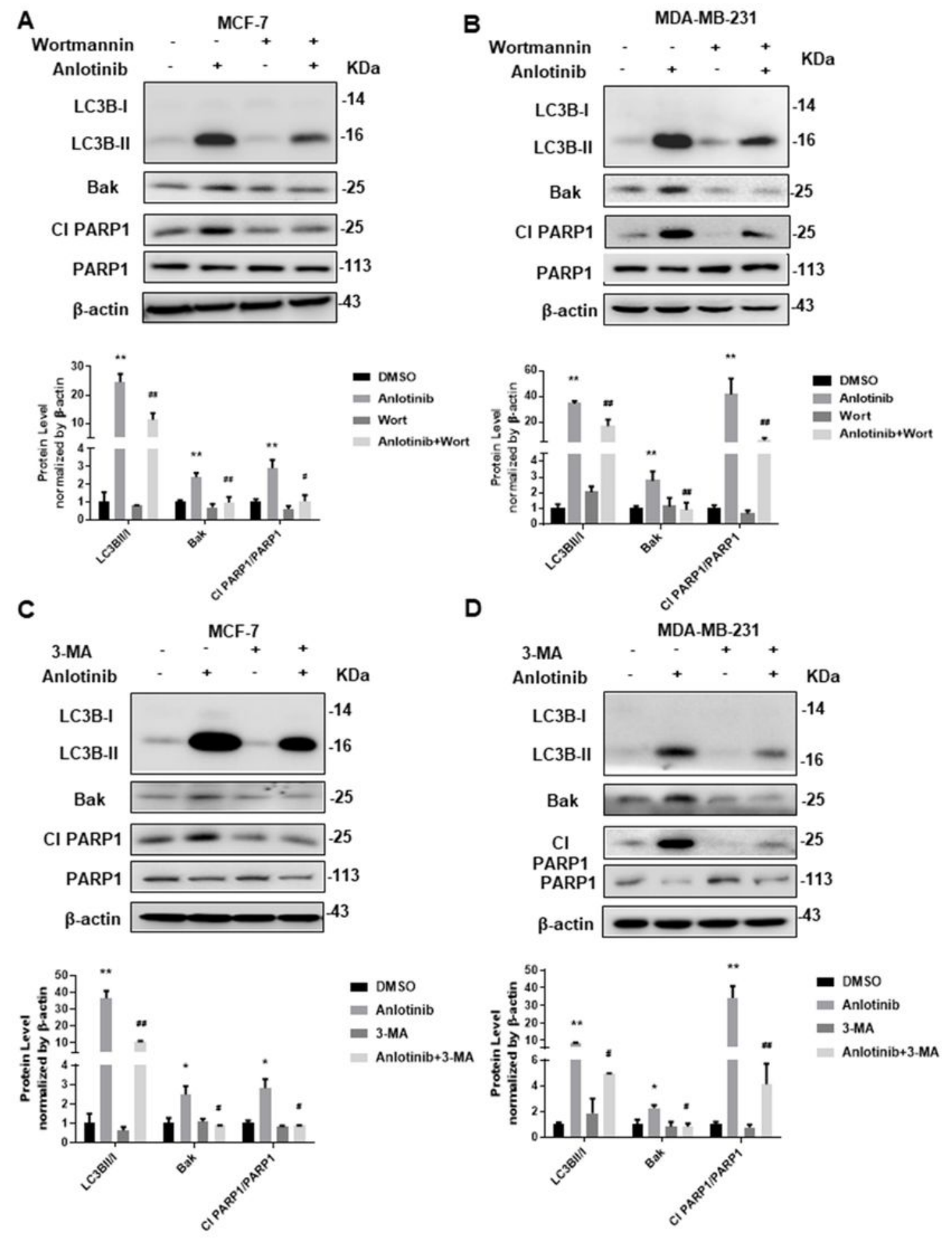

Figure 4 
Anlotinib induced apoptosis by promoting autophagy in MCF-7 and MDA-MB-231 cells with the mount of $10 \mu \mathrm{M}$ for $24 \mathrm{~h}$. A and B. The autophagy inhibitor wort protected BC cells from anlotinib-induced apoptosis. $C$ and $D$, The autophagy inhibitor 3-MA protected $B C$ cells from anlotinib-induced apoptosis. Western blot showing the ratio of LC3B II/LC3B I and the expression of apoptosis-associated proteins and the analysis. The data are shown as the mean \pm SD. ${ }^{*} p<0.05,{ }^{* *} p<0.01$ vs DMSO, $\# p<0.05, \# \# p<0.01$ vs Anlotinib, $n=3$.

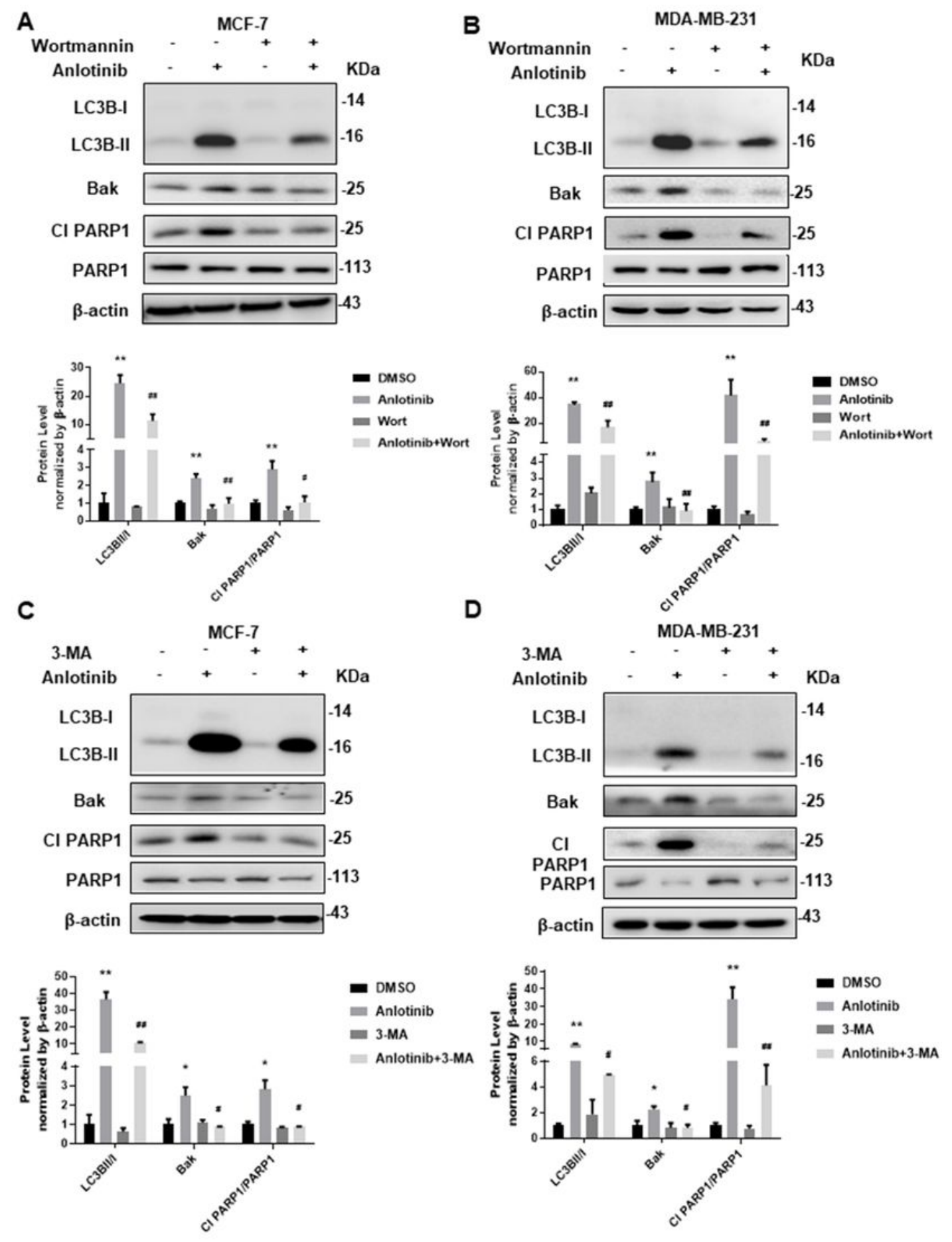


Anlotinib induced apoptosis by promoting autophagy in MCF-7 and MDA-MB-231 cells with the mount of $10 \mu \mathrm{M}$ for $24 \mathrm{~h}$. A and B. The autophagy inhibitor wort protected BC cells from anlotinib-induced apoptosis. $C$ and $D$, The autophagy inhibitor 3-MA protected $B C$ cells from anlotinib-induced apoptosis. Western blot showing the ratio of LC3B II/LC3B I and the expression of apoptosis-associated proteins and the analysis. The data are shown as the mean \pm SD. ${ }^{*} p<0.05,{ }^{*} p<0.01$ vs DMSO, $\# p<0.05, \# \# p<0.01$ vs Anlotinib, $n=3$.

A
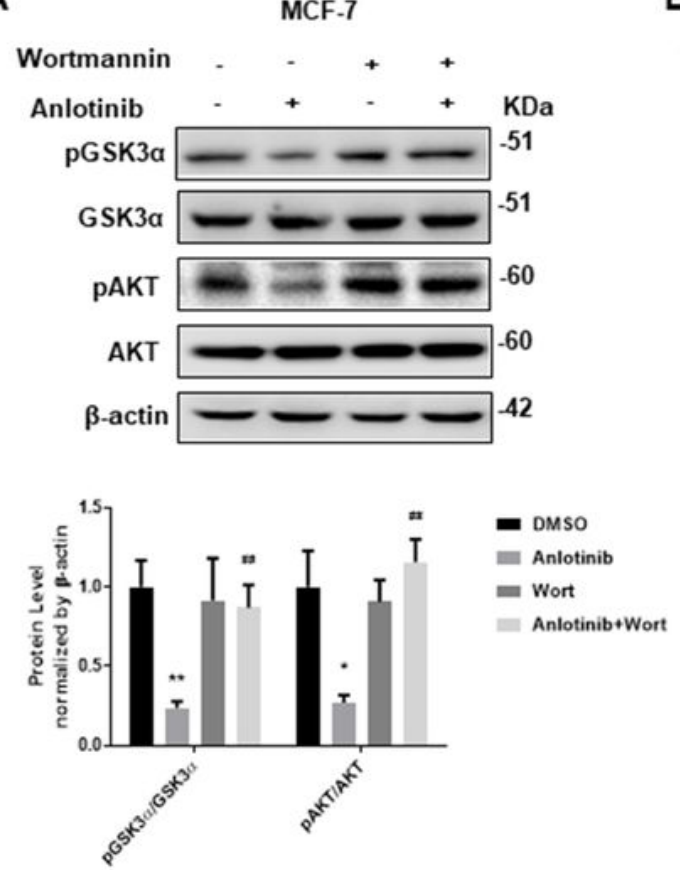

C

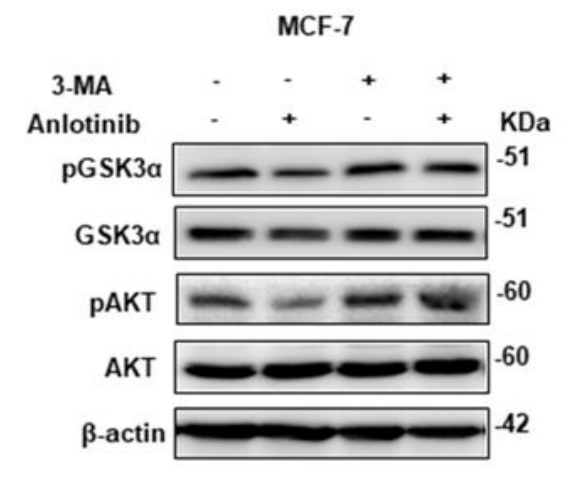

B
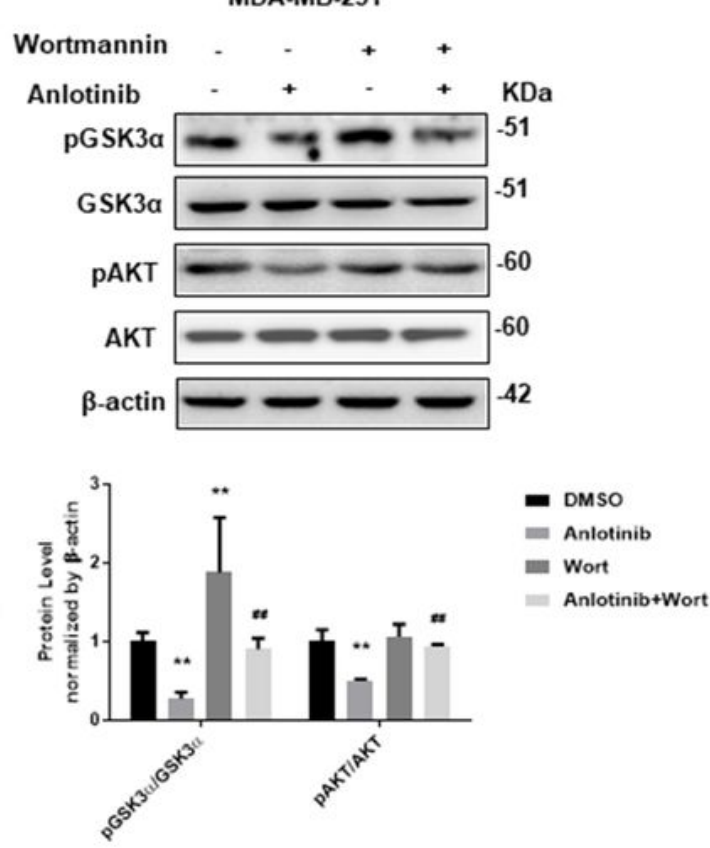

D

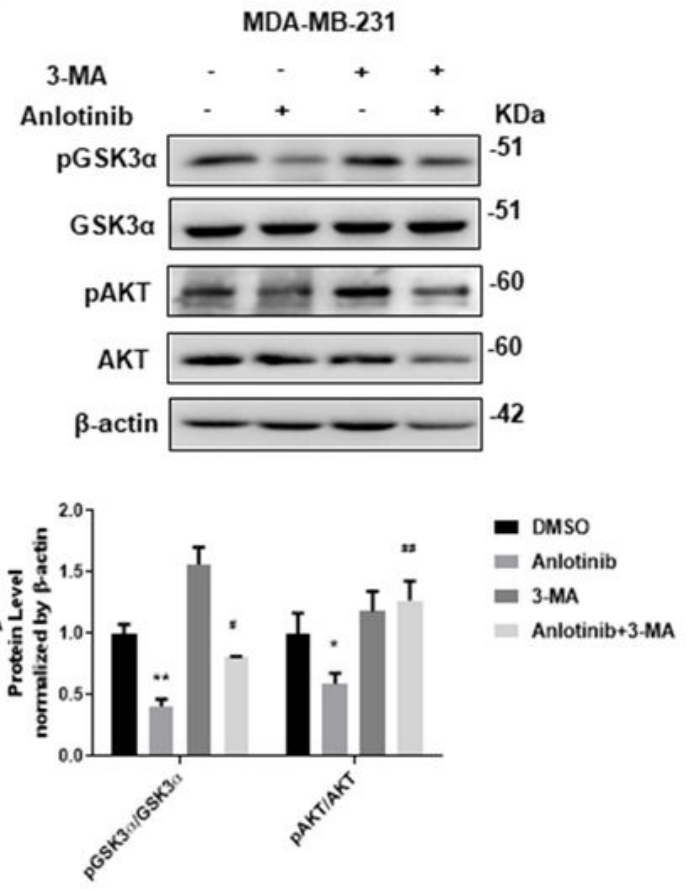


Anlotinib induced apoptosis by promoting autophagy by regulating the Akt/GSK-3a pathway. A-D, MCF-7 and MDA-MB-231 cells were pre-treated with the autophagy inhibitors wort and 3-MA before anlotinib treatment. The four groups were analysed by western blotting with total and phosphorylated antibodies for Akt and GSK-3a, and the data were normalized to $\beta$-actin. The data are shown as the mean \pm SD. *p $<$ $0.05,{ }^{* *} \mathrm{p}<0.01$ vs DMSO, $\# \mathrm{p}<0.05, \# \# \mathrm{p}<0.01$ vs Anlotinib, $\mathrm{n}=3$.

A
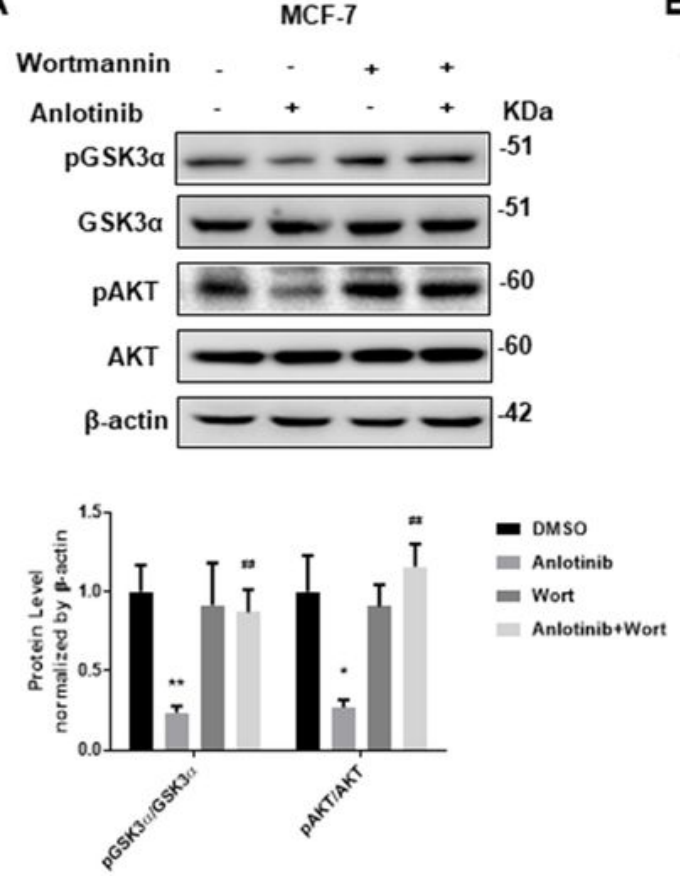

C

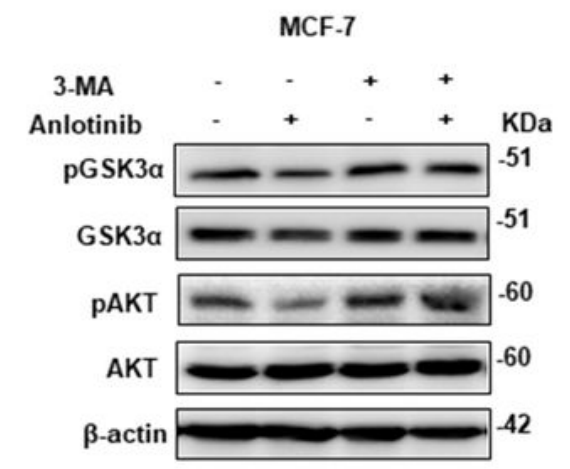

B
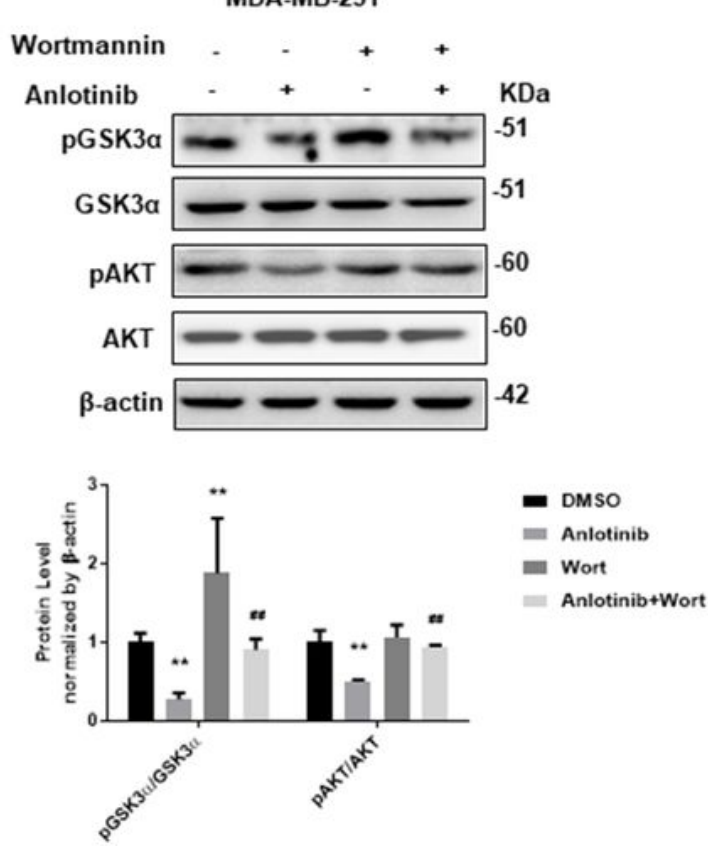

D

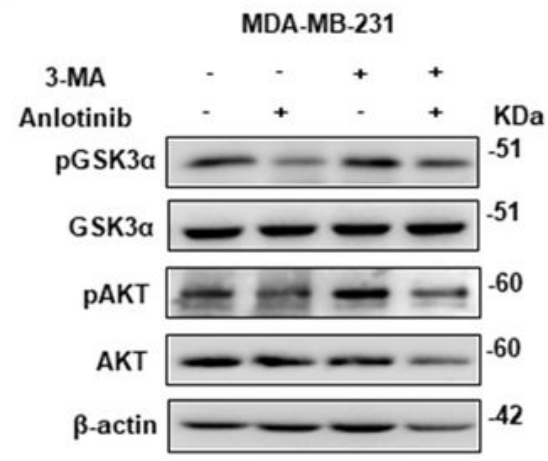

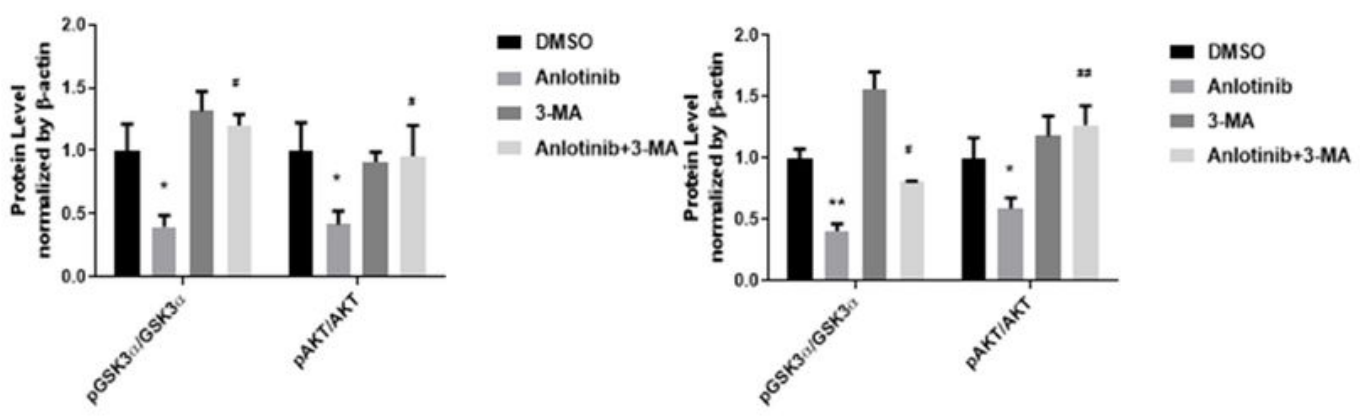

Figure 5 
Anlotinib induced apoptosis by promoting autophagy by regulating the Akt/GSK-3a pathway. A-D, MCF-7 and MDA-MB-231 cells were pre-treated with the autophagy inhibitors wort and 3-MA before anlotinib treatment. The four groups were analysed by western blotting with total and phosphorylated antibodies for Akt and GSK-3a, and the data were normalized to $\beta$-actin. The data are shown as the mean \pm SD. *p $<$ $0.05,{ }^{* *} \mathrm{p}<0.01$ vs DMSO, $\# \mathrm{p}<0.05, \# \# \mathrm{p}<0.01$ vs Anlotinib, $\mathrm{n}=3$.

A

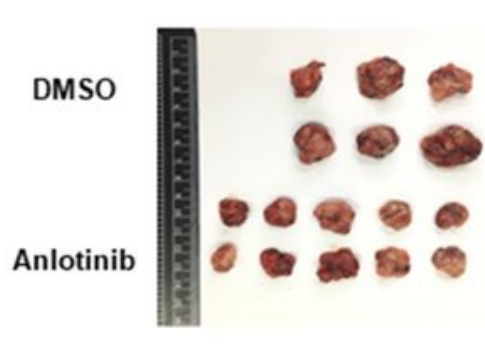

D

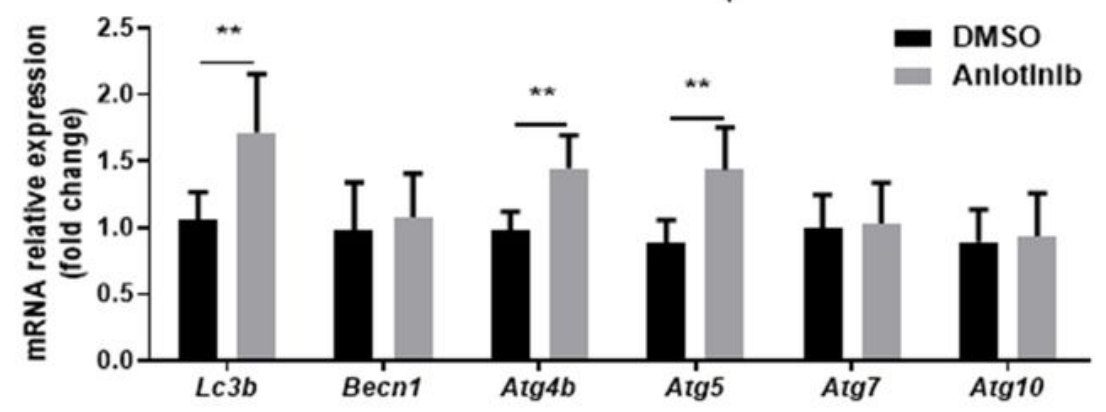

B

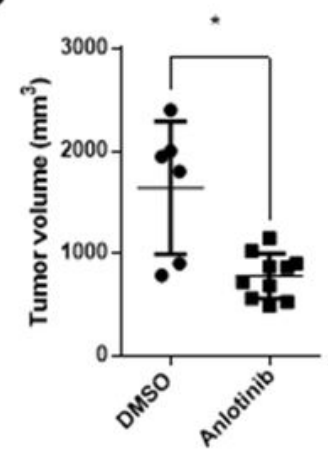

C

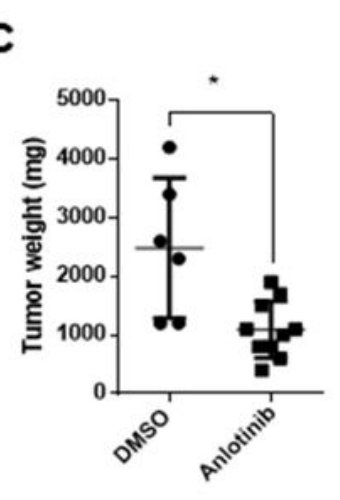


Anlotinib suppressed BC growth in vivo. A, Images of dissected tumours from C57BL/ 6 mice injected with AT-3 cells in the DMSO-treated group $(n=6)$ and the anlotinib-treated group $(n=10)$. B, Tumour volumes were decreased in the anlotinib group compared to those of the DMSO group. C, Tumour weights with anlotinib treatment were heavier than those without anlotinib treatment. D. The mRNA level of autophagyassociated molecules was increased markedly in the anlotinib-treated tumour tissues. $E$ and $F$. The ratio of LC3B II/LC3B I and the expression level of Bcl-2, Cyclin D1 and Cl-Caspase 3 were detected by western blotting and analysis. G. Ki67 immunohistochemistry staining of tumour sections from DMSO and anlotinib mice. Scale bars: $50 \mu \mathrm{m}$. The data are shown as the mean \pm SD. ${ }^{*} p<0.05,{ }^{*} p<0.01$ vs DMSO. 
A

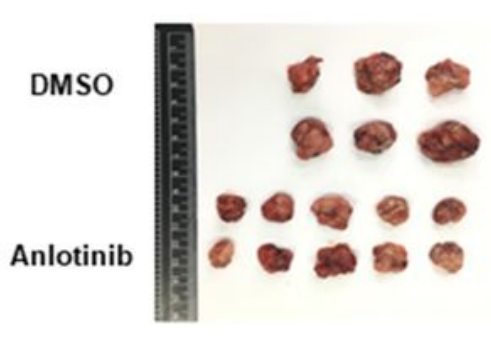

B

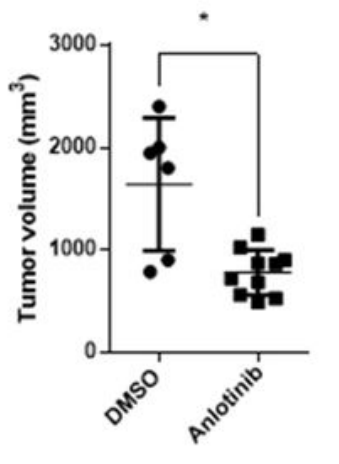

C

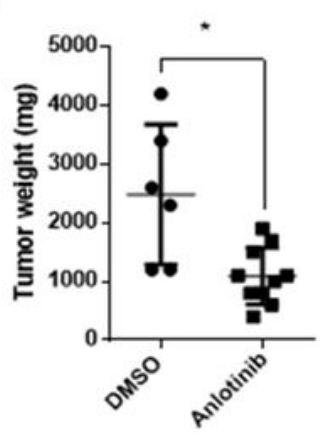

DMSO

Anlotinlb
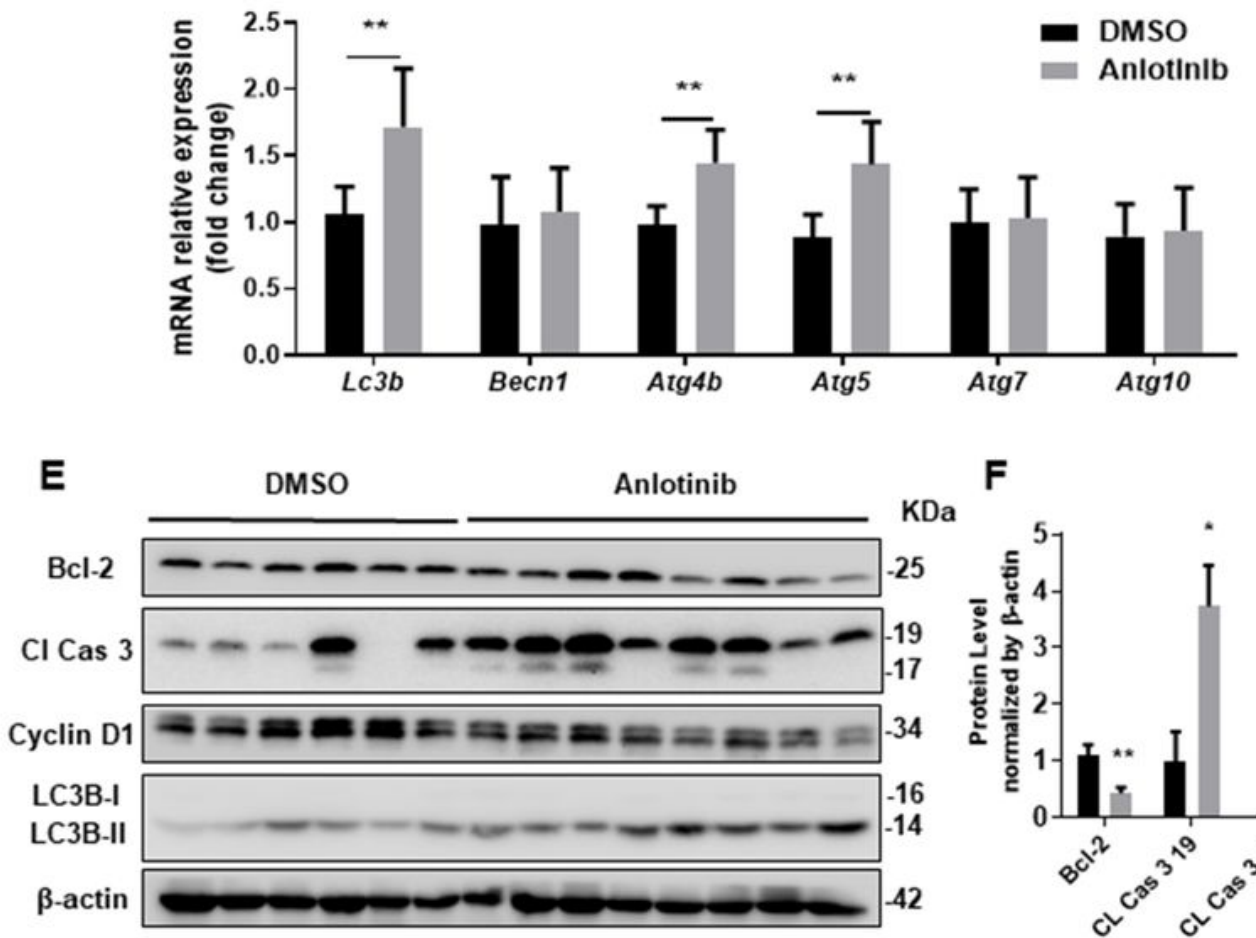

$F$

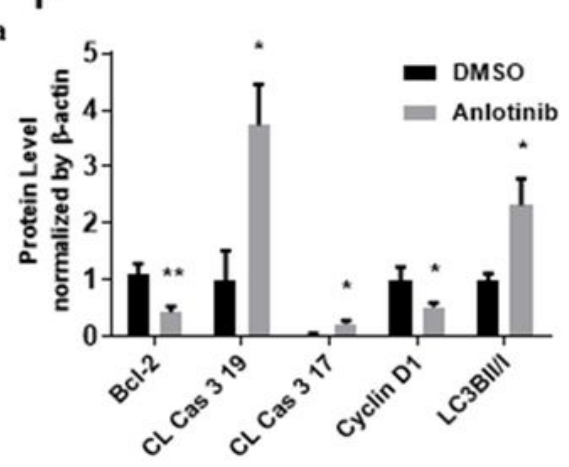

G

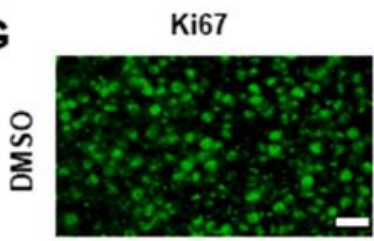

DAPI

Merge
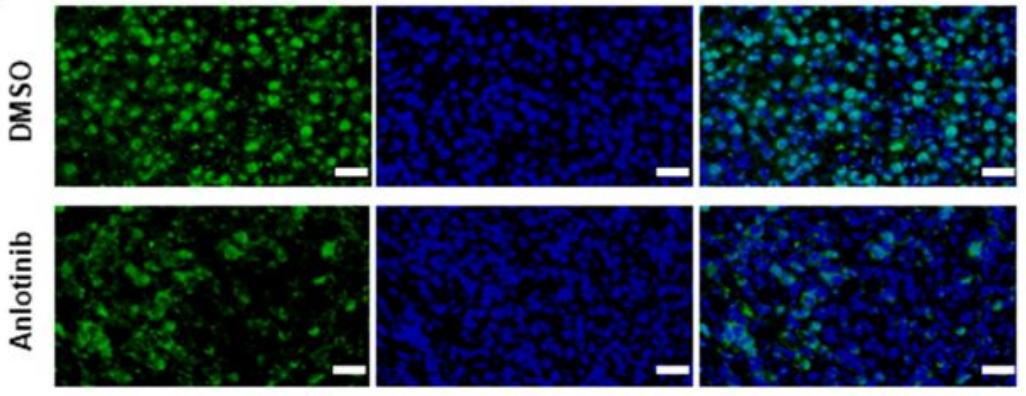

\section{Figure 6}

Anlotinib suppressed BC growth in vivo. A, Images of dissected tumours from C57BL/ 6 mice injected with AT-3 cells in the DMSO-treated group $(n=6)$ and the anlotinib-treated group $(n=10)$. B, Tumour volumes were decreased in the anlotinib group compared to those of the DMSO group. C, Tumour weights with anlotinib treatment were heavier than those without anlotinib treatment. D. The mRNA level of autophagyassociated molecules was increased markedly in the anlotinib-treated tumour tissues. $\mathrm{E}$ and $\mathrm{F}$. The ratio 
of LC3B II/LC3B I and the expression level of Bcl-2, Cyclin D1 and Cl-Caspase 3 were detected by western blotting and analysis. G. Ki67 immunohistochemistry staining of tumour sections from DMSO and anlotinib mice. Scale bars: $50 \mu \mathrm{m}$. The data are shown as the mean \pm SD. ${ }^{\star} p<0.05,{ }^{\star \star} p<0.01$ vs DMSO.

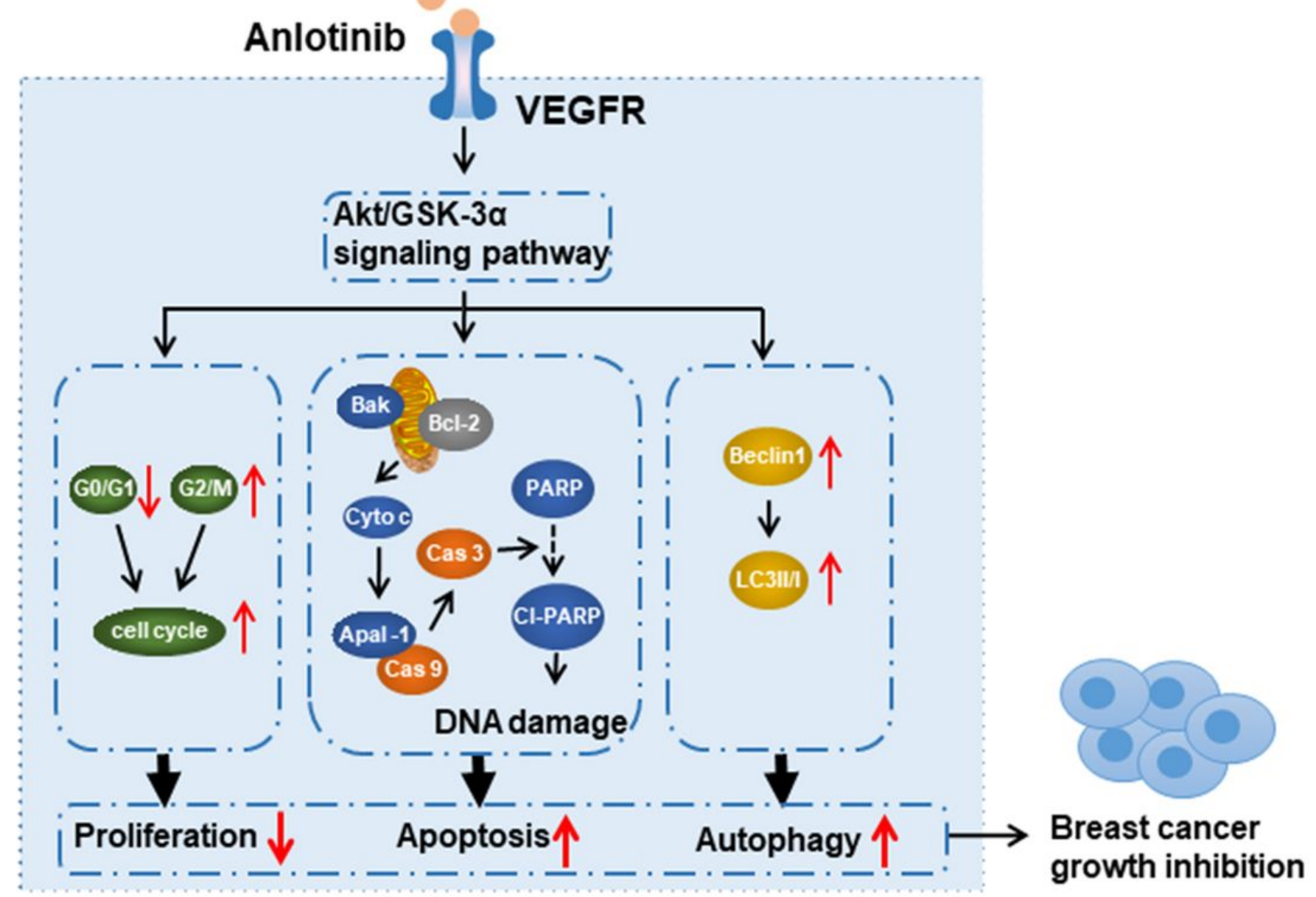

Figure 7

The mechanism by which anlotinib exerted its anticancer effect in BC. 


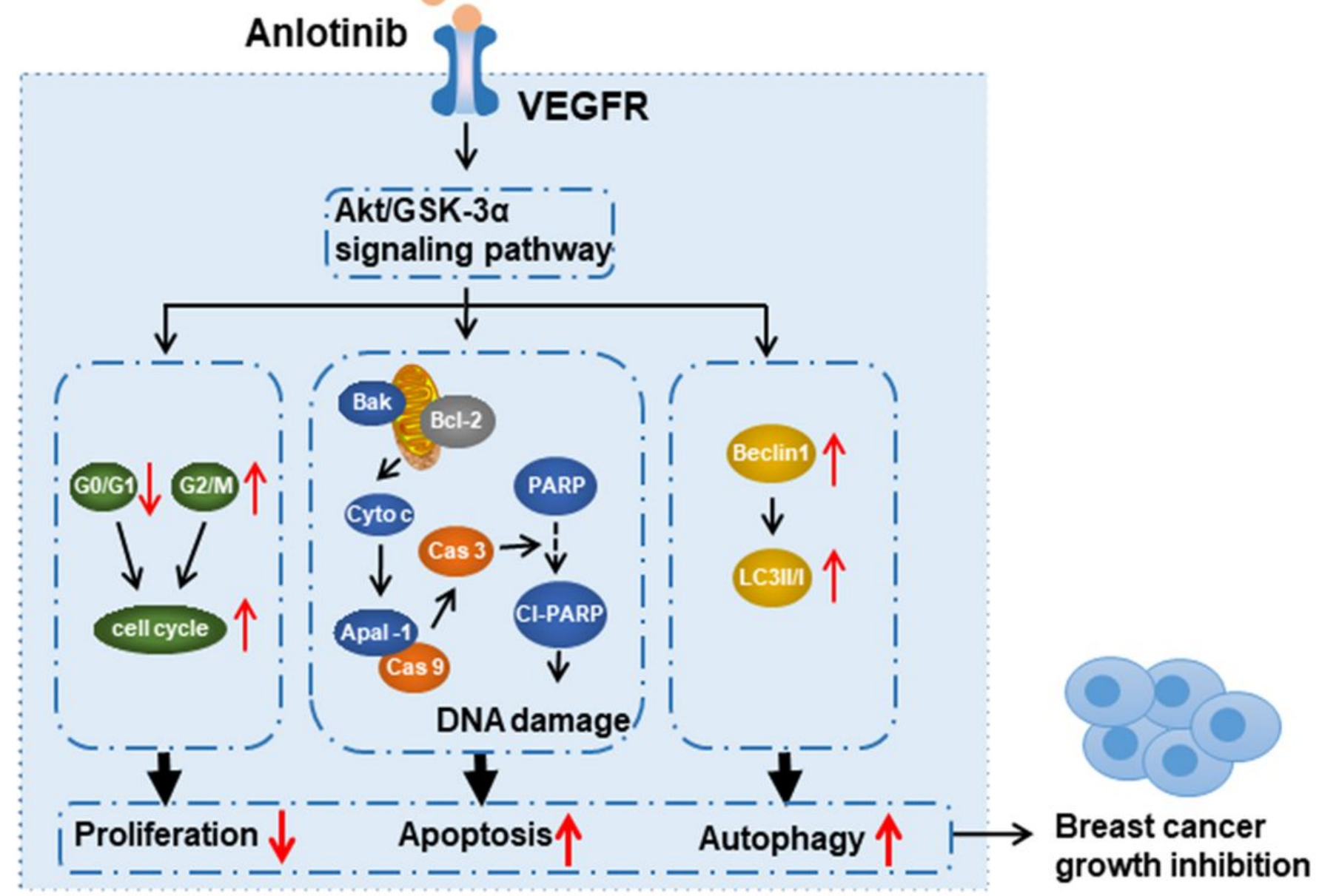

Figure 7

The mechanism by which anlotinib exerted its anticancer effect in BC.

\section{Supplementary Files}

This is a list of supplementary files associated with this preprint. Click to download.

- supportinginformation.docx

- supportinginformation.docx 